International Journal of Modern Physics B

(C) World Scientific Publishing Company

\title{
LONG-TIME RELAXATION ON SPIN LATTICE AS A MANIFESTATION OF CHAOTIC DYNAMICS
}

\author{
BORIS V. FINE \\ Max Planck Institute for the Physics of Complex Systems, Noethnitzer Str. 38 \\ 01187 Dresden, Germany \\ fine@mpipks-dresden.mpg.de \\ Received Day Month Year \\ Revised Day Month Year
}

\begin{abstract}
The long-time behavior of the infinite temperature spin correlation functions describing the free induction decay in nuclear magnetic resonance and intermediate structure factors in inelastic neutron scattering is considered. These correlation functions are defined for one-, two- and three-dimensional infinite lattices of interacting spins both classical and quantum. It is shown that, even though the characteristic timescale of the long-time decay of the correlation functions considered is non-Markovian, the generic functional form of this decay is either simple exponential or exponential multiplied by cosine. This work contains (i) summary of the existing experimental and numerical evidence of the above asymptotic behavior; (ii) theoretical explanation of this behavior; and (iii) semi-empirical analysis of various factors discriminating between the monotonic and the oscillatory long-time decays. The theory is based on a fairly strong conjecture that, as a result of chaos generated by the spin dynamics, a Brownian-like Markovian description can be applied to the long-time properties of ensemble average quantities on a non-Markovian timescale. The formalism resulting from that conjecture can be described as "correlated diffusion in finite volumes."
\end{abstract}

Keywords: Chaos; spin dynamics; free induction decay.

\section{Introduction}

One of the theoretical challenges associated with strongly interacting many-body systems is how to calculate the fast relaxation that takes place on a timescale of the order of the dynamic memory time of individual particles. Generic problems of this kind do not have enough small parameters to be solved in a controllable approximation. In such a situation, it is the intuitive concept of chaotic motion that, nevertheless, preserves the hope that relatively simple theories can make quantitative predictions on the fast timescale. An important question in this context is: If the system is, indeed, chaotic, does it lead to the properties of the fast relaxation, which are independent of the specific form of the inter-particle interaction?

In this work $^{1}$, we consider systems of interacting spins-both classical and quantum - and focus on the functional form of the long-time behavior of certain fast decaying correlation functions. We point to experimental and numerical evi- 
dence that the functional form in question is surprisingly simple in both classical and spin $1 / 2$ systems. We then produce an extensive argument asserting that even when conventional Markovian assumptions are not supposed to apply, it is still possible to justify quite an unusual diffusion description of the spin systems based on the assumption of chaotic dynamical mixing. In the framework of such a description, the observed functional form of the long-time behavior appears as a generic property of the correlation functions considered.

\subsection{Quantity of interest}

We study the infinite temperature correlation functions defined on an infinite spin lattice as:

$$
G(t)=\left\langle S_{0}^{\mu}(t) \sum_{n} \cos \left(\boldsymbol{q} \cdot \boldsymbol{r}_{n}\right) S_{n}^{\mu}(0)\right\rangle,
$$

where $S_{n}^{\mu}$ is either the classical projection or the quantum spin operator representing the $\mu$ th $\left(x, y\right.$ or $z$ ) spin component on the $n$th lattice site; $\boldsymbol{r}_{n}$ is the translation vector between the zeroth and the $n$th sites; and $\boldsymbol{q}$ is a wave vector corresponding to a spatial period commensurate with the lattice periodicity. The lattice can have any Bravais structure in one, two or three dimensions. Each spin on the lattice interacts with a finite number of neighbors according to the Hamiltonian

$$
\mathcal{H}=\sum_{k<n}\left[J_{k n}^{x} S_{k}^{x} S_{n}^{x}+J_{k n}^{y} S_{k}^{y} S_{n}^{y}+J_{k n}^{z} S_{k}^{z} S_{n}^{z}\right]
$$

where $J_{k n}^{\mu}$ are coupling constants. These constants must be such that Hamiltonian (2) is invariant with respect to lattice translations. Formula (1) distinguishes the zeroth spin from other spins only for the convenience of the later discussion. We shall also use variable $S$ with no indices to refer to the absolute values of individual spins.

Given the Hamiltonian (2), the timescale of individual spin motion, referred to below as "fast," "short," or "mean free time," is well-represented by the time $\tau$ defined as

$$
\tau=\left[S^{2} \sum_{n, \mu} J_{k n}^{\mu 2}\right]^{-1 / 2} .
$$

Unless special reasons exist, this timescale characterizes both the short- and the long-time decay of $G(t)$.

Another fact worth mentioning is that, as a result of the time reversibility of the spin dynamics, $G(t)$ is an even function of $t$, which, in particular, implies $\left.\frac{d G}{d t}\right|_{t=0}=0$.

In the context of inelastic neutron scattering, the correlation functions (1) are referred to as intermediate structure factors ${ }^{2}$. If $q=0$, Eq.(1) can also represent the free induction decay in nuclear magnetic resonance $(\mathrm{NMR})^{3},{ }^{4}$. From the viewpoint of practical implications, the subject of this work is more relevant to NMR, because NMR deals almost exclusively with high temperatures (on the scale of nuclear spin energies). 


\subsection{Nonequilibrium interpretation}

The infinite-temperature limit offers a unique advantage, which will be important for our subsequent treatment. Namely, the correlation function $G(t)$ can be considered as proportional to the polarization of the zeroth spin $\left\langle S_{0}^{\mu}(t)\right\rangle_{\rho(0)}$ averaged with the initial distribution function (or quantum density matrix)

$$
\rho(0) \simeq \exp \left[\beta_{0} \sum_{n} \cos \left(\boldsymbol{q} \cdot \boldsymbol{r}_{n}\right) S_{n}^{\mu}(0)\right],
$$

provided the inverse temperature $\beta_{0}$ is very small, and only the first order of $\beta_{0}$ is kept in the expansion of $\left\langle S_{0}^{\mu}(t)\right\rangle$. In other words, we are dealing with the decay of the average spin polarization on the zeroth lattice site, given weak initial polarization on each site proportional to $\cos \left(\boldsymbol{q} \cdot \boldsymbol{r}_{n}\right)$. For the sake of physical interpretation, one can imagine that, at $t<0$, the Hamiltonian (2) was switched off, and the spin system was equilibrated at very high temperature in an external field varying as $\cos \left(\boldsymbol{q} \cdot \boldsymbol{r}_{n}\right)$. Then, at $t=0$, the environment and the external field were switched off. Simultaneously, the Hamiltonian (2) was switched on, and the system started evolving to a new equilibrium, which, to the first order in $\beta_{0}$, corresponds to infinite temperature. This interpretation is visualized in Fig. 1 for classical spins.

\subsection{Theoretical goal}

In this work, we intend to show theoretically that generic long-time behavior of $G(t)$ has one of the following two functional forms: either

$$
G(t) \simeq e^{-\xi t}
$$

or

$$
G(t) \simeq e^{-\xi t} \cos (\eta t+\phi),
$$

where $\xi, \eta$ and $\phi$ are some constants about which we can only assert that, in general, the values of $\xi$ and $\eta$ are of the order of $1 / \tau$. It will follow from both the empirical evidence and our theory that $G(t)$ approaches the asymptotic form (5) or (6) after a time of the order of several $\tau$, i.e. sufficiently fast.

We define the "generic long-time behavior" as follows:

For a given lattice and for a given radius of interaction, there is a finite number of independent interaction coefficients necessary to specify the translationally invariant Hamiltonian (2). (For example, for the spin chain with the nearest neighbor interaction of form(2), one must specify only three coefficients $J^{x}, J^{y}$ and $J^{z}$.) If, we restrict the value of each independent interaction coefficient to a finite interval (the same for all of them) and, from that interval, pick the values of those coefficients randomly, then our claim is that, with probability 1 , the long-time behavior of the correlation functions (1) will have the functional form either (5) or (6).

The above definition implies that there can be infinitely many exceptions not exhibiting the long-time behavior $(5,6)$ - we cannot name all of them - but the overwhelmingly general rule is still given by Eqs. $(5,6)$. 
4 B. V. Fine

(a)
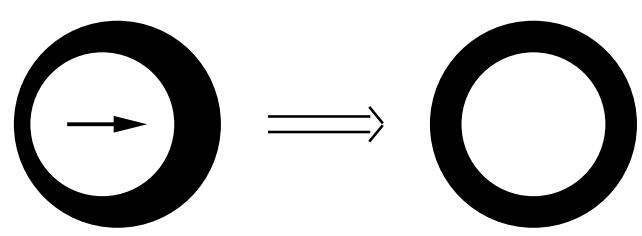

(b)

(I)

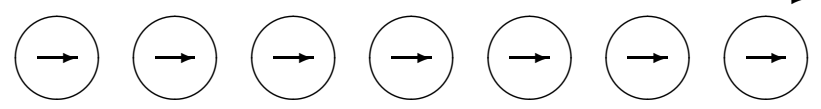

(II)
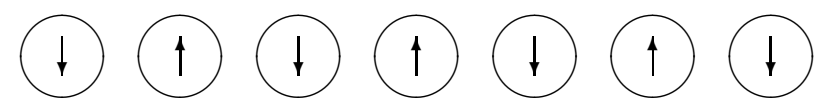

(III)
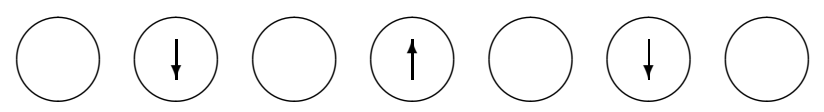

(IV)
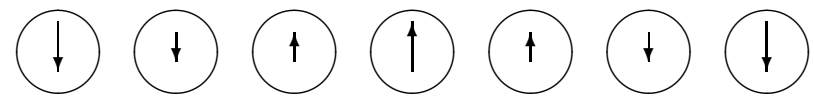

$-3$ $-2$

$-1$

0

1

2

3

Fig. 1. These pictures visualize the nonequilibrium problem corresponding to the initial probability distribution (4) for classical spins. Picture (a) represents an example of the initial and the final probability distributions of the zeroth classical spin. The tip of the spin vector moves on a spherical surface shown as a white disk. The thickness of the outer black layer around that disc represents the probability to find the spin oriented in the corresponding radial direction. The arrow in the middle of the white disc indicates the average spin polarization. Thus the problem is one of calculating the time dependence of the average spin polarization, given that the probability density on the spherical surface evolves from a weakly anisotropic distribution (left) to the completely isotropic infinite temperature distribution (right). Picture (b) shows examples of the initial conditions for fragments of infinite spin chains. The arrows indicate the direction and the relative size of the weak spin polarization at $t=0$. The numbers in the bottom line are the indices of the spin sites. Examples (I-IV) correspond to the calculation of the correlation functions of form (1), provided (I) $\mu \rightarrow x, q=0$; (II) $\mu \rightarrow z, q=\pi$; (III) $\mu \rightarrow z, q=\pi / 2$; (IV) $\mu \rightarrow z, q=\pi / 3$.

It is important to realize that, if the functional dependence $(5,6)$ is, indeed, generic, then this property is very likely related to the randomness generated by the spin dynamics. At the same time, the problem cannot be reduced to the Marko- 
vian paradigm of "a slow variable interacting with a fast equilibrating background" - the decay (5) or (6) occurs on the timescale of $\tau$, which is the fastest natural timescale of the problem. There would be no contradiction to the standard theory of Brownian-type motion (which is based on the above paradigm) if, on the timescale of $\tau$, the long-time decay of correlation functions (1) was described by a power law, Gaussian, or by some erratic functional form - different for different correlation functions. Therefore, whatever is the ultimate explanation of the long-time behavior $(5,6)$, it will certainly be a step beyond the standard theory of Brownian-type motion. In particular, formulas $(5,6)$ cannot be viewed as yet another example of the damped harmonic oscillator, because the standard treatment of the damped oscillator requires the separation of timescales between the slow oscillator and the quickly equilibrating microscopic motion in a heat bath.

It is also worth mentioning that the oscillations in Eq.(6) are neither related to a simple rotation nor produced by the equivalent of a simple spring potential. Below, we illustrate the non-trivial nature of this fact by considering the example of the NMR free induction decay, in which case the oscillations have actually been observed experimentally (see Section 2).

The NMR free induction decay is the decay of the macroscopic magnetization of nuclear spins under the influence of local fields fluctuating around the zero average value. When present, the oscillations of this decay are not the result of any kind of magnetization precession, i.e., at the moment when the free induction decay crosses zero, the magnetization of the system is equal to zero in all directions, and then, out of completely unpolarized state the magnetization reappears with the opposite sign. Such a fact is hard to understand intuitively, because, the value of the macroscopic magnetization appears to be a direct measure of the deviation from equilibrium, but, at the same time, normal intuition, which is supported by numerous examples of the Markovian limit, would suggest that, once at equilibrium, the system should not deviate from it (beyond the range of usual thermal fluctuations). It is then even harder to explain how the fluctuations of local fields cause the oscillations of the long-time decay to be periodic.

The importance of the long-time behavior $(5,6)$ should not be underestimated in light of the fact that there are many ways (memory functions, continued fractions, etc.) to produce such a behavior with very simple ingredients. It is precisely the justification of those ingredients that makes the whole issue intractable within any of those attempts.

In particular, a number of NMR-related theories ${ }^{5,8,6,7,9,11,10,12}$ would predict the long-time behavior given by Eqs. $(5,6)$. However, all of those theories struggle to achieve an effective approximation for the entire evolution of $G(t)$, of which the long-time part is not the most prominent one. As a result, the long-time functional dependence becomes a side effect of quite crude and uncontrollable assumptions adopted only for the sake of simplicity. Therefore, the long-time predictions of those theories remain doubtful. (See e.g. Ref. ${ }^{13}$.) In order to illustrate this point, in 
Appendix Appendix A we discuss the treatment of Borckmans and Walgraef ${ }^{6,7}$, who have made a particularly strong claim of the derivation of the long-time functional form $(5,6)$.

The theory to be presented in this work is unusual in one important respect. Namely, it leads to the definite result $(5,6)$ for the functional form of the longtime decay without giving the recipe for calculating the parameters describing that decay. Our viewpoint is that should the validity of Eqs. $(5,6)$ be shown in a modelindependent way, the approximate schemes can rely on such a result rather than derive it. As a consequence, the status of the approximate calculations would change from an uncontrollable extrapolation in the time domain to something more similar to interpolation. In order to obtain the necessary long-time parameters, it will be sufficient to compute the initial evolution of the correlation functions up to the point, where they are supposed to be describable by Eq.(5) or (6). In that scheme, the approximate analytical expressions for the initial evolution will not be required to have the asymptotic long-time form (5) or (6).

The existing approximation schemes $3,5,8,6,7,9,11,10,12,13,14$ are already quite good in describing the initial behavior of correlation functions, but, as far as the parameters of the long-time behavior are concerned, the a priori accuracy of predictions based on those schemes is yet to be established. (Our estimate is that it is not better than 20 per cent.)

Another unusual feature of our theory is that it relies on a relatively long chain of qualitative arguments. Though not a rigorous derivation, such a treatment allows us to address the questions, which were mostly ignored in the previous works. Namely:

Why is the long-time regime universal?

What makes that regime different from the initial regime?

Why does it have such a functional form?

Why are Eqs. $(5,6)$ primarily relevant to the $q$-dependent correlation functions (1) and not to the pair correlation functions of the form $\left\langle S_{k}^{\mu}(t) S_{n}^{\mu}(0)\right\rangle$ ?

What mechanism is responsible for the oscillations in Eq.(6)?

The answers to these questions will be given in Section 3.6.

A comment is now due on our use of term "chaos."

What we call "chaos" is a concept stronger than Boltzmann's "molecular chaos", because "molecular chaos" only postulates the irrelevance of fast decaying correlations to slow observables, while in this work we address precisely the issue of how those fast correlations decay. At the same time, our assumptions of chaos are formulated mostly in terms of the motion of a single particle, which is consistent with, but less restrictive than, the mathematical definition of chaos related to the exponential instabilities of trajectories in the phase space of the entire system. In particular, our treatment will not be altered if the assumed single particle properties result from taking the thermodynamic limit in the systems not quite complying with the 
mathematical definition of chaos.

We should also mention that there are apparent parallels between our treatment and the mathematical theory of Policott-Ruelle resonances in classical chaotic systems $^{15,16}$. However, we are not aware of any definite mathematical result applicable to spin lattices with time-independent translationally invariant Hamiltonians, in particular, in the quantum case. One of the advantages of our approach is that it allows the quantum case to be addressed directly, i.e. without any reference to the classical limit. Recently, Prosen has arrived to the same conclusion about the role of the Policott-Ruelle resonances by considering the decay of correlation functions of kicked Ising chain of spins $1 / 2^{17,18}$.

The plan of the rest of this paper is the following: In Section 2 we summarize the empirical evidence of the long-time behavior $(5,6)$. In Section 3 we develop the theory of the long-time relaxation. Finally, in Section 4, we discuss various factors, which discriminate between the monotonic regime (5) and the oscillatory regime (6).

\section{Empirical evidence}

Before reviewing the existing experimental and numerical evidence for the long-time behavior (5) and (6), we recall that the characteristic decay time of $G(t)$ is expected to be of the order of $\tau$ given by Eq.(3). At the same time, the characteristic longtime behavior does not become pronounced until after several $\tau$, i.e. until $G(t)$ has fallen to a relatively small value. Yet, in order to make definite conclusions about the long-time behavior, one has to go orders of magnitude below the initial value of $G(t)$. This task is extremely challenging both numerically and experimentally. In particular, it is certainly beyond the reach of the present day inelastic neutron scattering experiments.

There exist two pieces of evidence unambiguously showing the oscillatory longtime behavior (6) in spin 1/2 systems: (i) experiments on NMR free induction decay in $\mathrm{CaF}_{2}$ by Engelsberg and Lowe ${ }^{19}$, and (ii) the results of numerical diagonalization of spin $1 / 2$ chains by Fabricius, U. Löw and J. Stolze ${ }^{20}$.

In the first case, the underlying theoretical problem involves a simple cubic lattice of spins $1 / 2\left({ }^{19} \mathrm{~F}\right.$ nuclei) coupled by a truncated magnetic dipolar interaction ${ }^{4}$, which has the form (2) with $J_{k n}^{z}=-2 J_{k n}^{x}=-2 J_{k n}^{y} \simeq \frac{\left(1-3 \cos ^{2} \vartheta\right)}{\left|\boldsymbol{r}_{k}-\boldsymbol{r}_{n}\right|^{3}}$, where $\vartheta$ is the angle between $\left(\boldsymbol{r}_{k}-\boldsymbol{r}_{n}\right)$ and the $z$-axis. The free induction decay is then described by Eq.(1), with $q=0$ and $\mu \rightarrow x$. In the context of the experiment, the above quantity is proportional to the decay of the average spin polarization in the Larmor rotating reference frame provided the $z$-axis corresponds to the direction of the strong external magnetic field. This implies that the values of the interaction coefficients can be changed by varying the direction of the external field with respect to the crystal lattice.

All three free induction decays reported in Ref. ${ }^{19}$ are reproduced in Fig.2(a). 
The plots are labelled by the crystal direction of the external field.

It should be mentioned that there is no material other than $\mathrm{CaF}_{2}$, where the inter-nuclear spin-spin interaction is so well-known and so well-isolated from other microscopic factors, and where, at the same time, the free induction decay is measured with sufficient accuracy. In a less refined setting, the oscillating tails of free induction decays with nodes, which, at longer times, become equally spaced have also been observed by Metzger and Gaines ${ }^{21}$ in solidified mixtures of molecular hydrogen and deuterium.

The second piece of evidence for the long-time behavior (6) comes from the numerical diagonalization of the chains (rings) of sixteen spins $1 / 2$ coupled by the nearest-neighbor interaction of form (2), with the restriction $J_{n, n+1}^{x}=J_{n, n+1}^{y}$. These chains are known as $X X Z$ chains. Three correlation functions of form (1) have been presented in Ref. ${ }^{20}$, and are reproduced in Fig. 2(b). Each of those correlation functions is characterized by the following parameters entering Eqs.(1, 2): $q=\pi$ (in the units of inverse chain spacing), and $J_{n, n+1}^{x}=J_{n, n+1}^{y}=J$, where $J$ is an auxiliary variable. Other parameters are: (I) $J_{n, n+1}^{z}=J$, any value of $\mu$; (II) $J_{n, n+1}^{z}=J \cos (0.3 \pi), \mu \rightarrow z$; (III) $J_{n, n+1}^{z}=J \cos (0.3 \pi), \mu \rightarrow x$.

In principle, it is an open question to what extent the the spin $1 / 2 X X Z$ chains with $J_{n, n+1}^{z} \neq 0$ display generic properties ${ }^{22}$, because, on one hand, they are known to be integrable, but, on the other hand, if attempted, the actual solution is so complex that the explicit form of the correlation functions of interest has never been obtained. As far as the infinite temperature correlation functions are concerned, the numerical study of those chains ${ }^{2022}$ did not reveal anything qualitatively unusual. Therefore, for now, since not much data on the long-time behavior of these functions exist, it is reasonable to take the data on the spin $1 / 2 X X Z$ chains as a strong hint for the generic nature of Eq.(6). However, in the future, if more evidence for the long-time behavior $(5,6)$ in generic spin systems emerges, the logic can be turned around, and the existence of such behavior in the spin $1 / 2 X X Z$ chains can be considered as a piece of evidence proving that the properties of those integrable chains are, at least, partially generic.

We are not aware of any data on quantum spin systems with time-independent Hamiltonians which would definitely support the monotonic exponential long-time behavior (5), when no separation of timescales is present in the problem. However, it is common knowledge in the field of NMR, that monotonic exponential longtime behavior of free induction decay occurs even more frequently than oscillatory behavior. Simple exponential decay of correlation functions has also be observed by Prosen in the numerical studies of a quantum spin system with time-dependent Hamiltonian (kicked Ising chain of spins $1 / 2)^{17,18}$.

Monotonic exponential decay is, in a sense, less surprising, given that at least it is expected in the situation when the separation of timescales is present, i.e. when $G(t)$ evolves much slower than a typical microscopic variable. An example of such a situation is the problem of "exchange narrowing" 23,24 , in which case, the dominating part of the Hamiltonian (2) has the Heisenberg form $\left(J_{k n}^{x}=J_{k n}^{y}=J_{k n}^{z}\right)$, and the 
(a) NMR free induction decay in $\mathrm{CaF}_{2}$

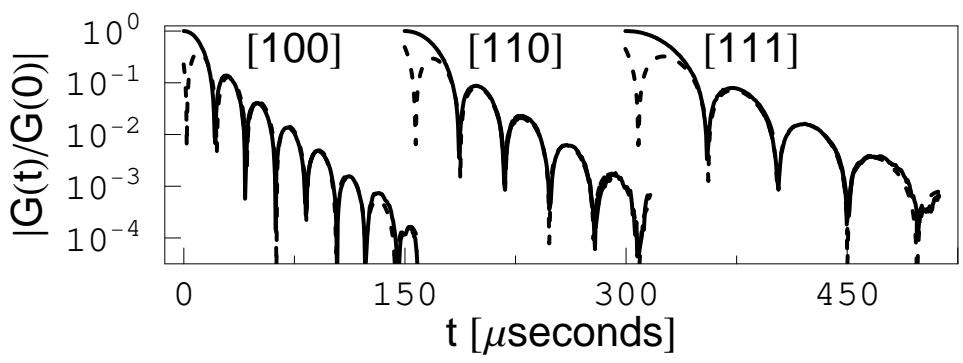

(b) Chains of spins $1 / 2$

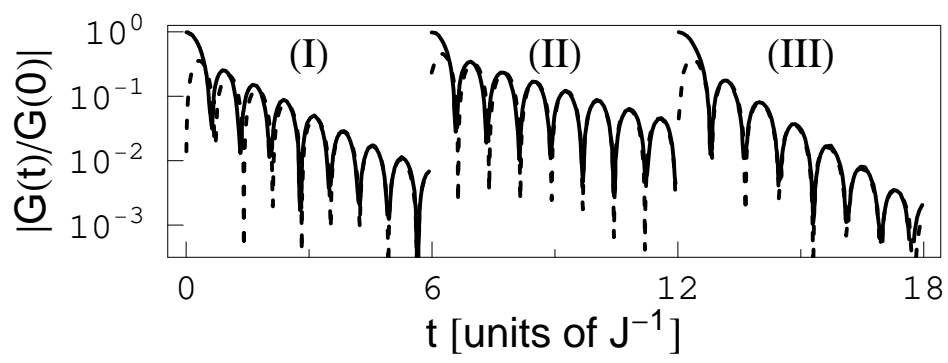

(C) Lattices of classical spins
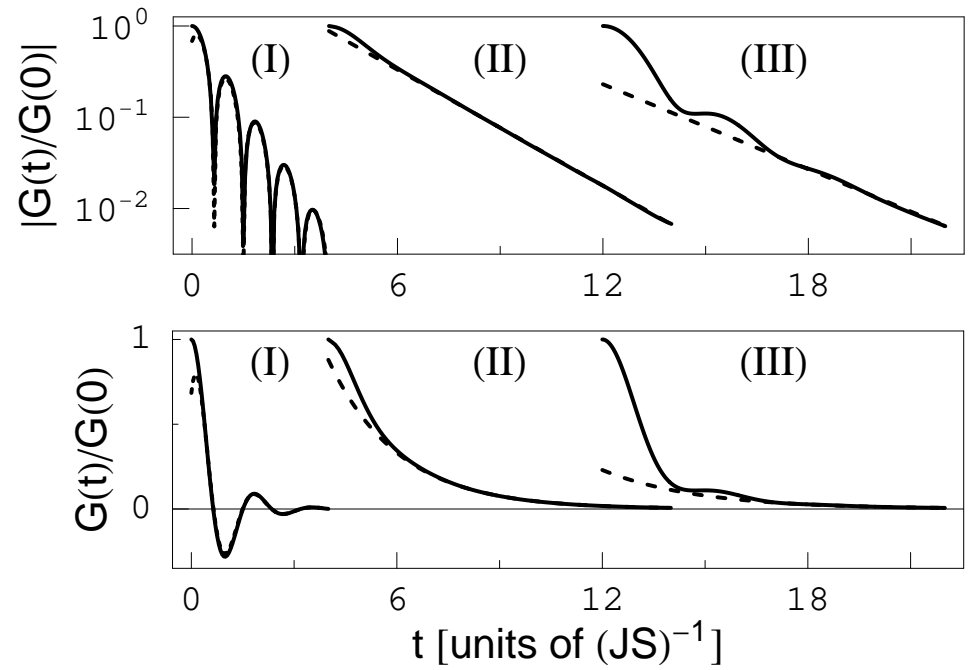

Fig. 2. Experimental (a) and numerical (b,c) evidence of the long-time behavior $(5,6)$. The solid lines represent the data reproduced from the following sources: (a) Ref. ${ }^{19}$; (b) Ref. ${ }^{20}$; (c) Ref. ${ }^{26}$. The dashed lines represent long-time fits of the form (5) or (6). In each frame, the origins of the middle and the right plots are displaced along the time axis. In all frames, except for the last one, the absolute values of $G(t)$ are presented on a logarithmic scale, which leads to cusps when $G(t)$ actually crosses zero. For reference, the data set (c) set is also presented on a direct (nonlogarithmic) scale with no absolute value taken. The details describing each of the plots are given in the text. 
correlation function of interest is given by Eq.(1) with $q=0$. This correlation function does not decay unless the Hamiltonian contains small corrections to the Heisenberg interaction. The exponential decay of the slow correlation functions or, more precisely, the Lorentzian shape of their Fourier transforms has been verified experimentally ${ }^{25}$ in several quantum spin systems exhibiting exchange narrowing.

Motivated by the spin $1 / 2$ problems and by the theory to be presented below, we have recently performed numerical simulations of classical spin systems ${ }^{26}$. The simulations generated a wide spectrum of data covering different dimensions, interactions and wave vectors - all entirely consistent with the long-time behavior $(5,6)$. Three of the nine correlation functions computed in Ref. ${ }^{26}$ are reproduced in Fig. 2(c). Two of them demonstrate the monotonic long-time behavior (5) missing in the previous examples. Each of the correlation functions presented in Fig. 2(c) corresponds to $\mu \rightarrow x$ in Eq.(1), and has been computed for the nearest-neighbor version of Hamiltonian (2). The remaining details (specific for each plot) are: (I) three-dimensional cubic lattice, $\boldsymbol{q}=(0,0,0), J_{k n}^{x}=0, J_{k n}^{y}=-J, J_{k n}^{z}=J$; (II) two-dimensional square lattice, $\boldsymbol{q}=(\pi / 2, \pi), J_{k n}^{x}=1.2 J, J_{k n}^{y}=-0.2 J, J_{k n}^{z}=J$; (III) one-dimensional chain, $q=0, J_{k n}^{x}=1.2 J, J_{k n}^{y}=-0.3 J, J_{k n}^{z}=J$.

It thus appears that the long-time behavior represented by Eqs. $(5,6)$ is very real. Its simplicity, uncharacteristic of the timescale involved, calls for a theoretical explanation, which is the subject of the rest of this work.

\section{Theory of the long-time relaxation}

\subsection{Outline}

Our theory is to be developed according to the following plan: First, in Section 3.2, we introduce the classical case but then digress and, in Section 3.3, analyze one rarely discussed piece of empirical knowledge related to the Markovian description. This analysis generates Conjecture I, on which the rest of the theory is built. Section 3.3 is quite long, but, in fact, reading only the formulation of Conjecture I is sufficient to proceed to Sections 3.4 and 3.5. In Section 3.4, we return to the treatment of classical spins and, in Section 3.5, extend that treatment to quantum spins. Although the classical limit of the quantum problem will not be taken, the treatment of quantum spins will be developed by analogy with the classical spins. Therefore, reading the classical section is essential to understanding of the quantum section. Finally, we summarize the theory in Section 3.6.

\subsection{Classical spins - long-time assumption}

We shall treat the classical case from the viewpoint of the nonequilibrium interpretation presented in Section 1.2 and illustrated in Fig. 1. Our attention will be focused on the one-particle probability distributions of the spin orientations.

The evolution of each classical spin vector can be represented as a trajectory of its tip on a spherical surface. Describing the ensemble of all possible trajectories of 
a given spin, we assume chaotic mixing of the following kind:

On the scale characterized by the mean free time $\tau$ given by Eq.(3) and by the corresponding mean free path (of the order of the radius of the sphere), (i) each trajectory loses the memory of its initial position; (ii) the set of all possible trajectories starting from any arbitrary small surface element disperses over the entire spherical surface in a random manner; and (iii) the statistics of the trajectory patterns of this set becomes representative of the statistics of the whole ensemble of one-spin trajectories.

The above assumption of chaotic mixing does not require the statistical properties of every one-spin trajectory to be representative of the properties of the whole ensemble. This detail signifies the difference between mixing and ergodicity. Following Krylov ${ }^{27}$, we assume that ergodicity is not a necessary condition for relaxation to the equilibrium. The estimate of the mean free time in this assumption by formula (3) is how the infinite temperature condition enters our treatment. At finite temperatures, the mean free time should be longer, and may even become infinite, because of the equilibrium correlations between the orientations of different spins.

If a point-like particle moves under similar conditions on an infinite plane, it would be appropriate to apply the theory of Brownian motion on a scale much greater than the mean free path. The problem is that in our case the typical mean free path is of the order of the size of the sphere. Therefore, we are interested in an accurate description on the scale of the order of the mean free path and smaller.

We now turn to a general discussion that motivates one additional assumption.

\subsection{Markovian description for the ensembles of trajectories}

The standard microscopic theory of Brownian motion is Markovian, i.e. it employs processes possessing no memory of the past state. It is always emphasized (at least in the physics literature) that such a description is justified only on a timescale much greater than the mean free time.

Contrary to the above point, we shall argue that, as far as ensemble averaging is concerned, the adequacy of the commonly accepted Markovian descriptions typically propagates to much shorter time scales, and it is only the initial short-time behavior of a typical theoretical quantity that is not Markovian.

\subsubsection{Exponential decay}

The inapplicability of the Markovian description to short (ballistic) timescales is frequently demonstrated by presenting an example of the nearly exponential decay exhibited by an autocorrelation function having the general form

$$
R(t)=\langle X(t) X(0)\rangle .
$$

This function characterizes the equilibrium fluctuations of some variable $X$ in some macroscopic system (not specified here). The variable $X$ should evolve much slower than the rest of the variables describing the same system. In equilibrium, $\langle X\rangle=0$, 
which implies that $R(\infty)=0$. (An example of such a situation is the exchange narrowing problem mentioned in Section 2.)

The following assumptions with respect to $R(t)$ are usually appropriate (at least the "usual wisdom" suggests so):

Assumption 1: The equilibrium correlation function $R(t)$ can be equivalently considered as characterizing the relaxation of the slow average quantity $\langle X(t)\rangle_{\rho_{R}(0)}$ starting from a very small initial value $\langle X(0)\rangle_{\rho_{R}(0)}$ and decaying to the equilibrium zero value. The notation \langle\rangle$_{\rho_{R}(0)}$ implies that the averaging should be taken over all possible evolutions of the entire system weighed by the probability of the initial conditions denoted here as $\rho_{R}(0)$. The small deviation of $\rho_{R}(0)$ from the equilibrium distribution function should be proportional to $X(0)$.

As explained in Section 1.1, as far as the infinite temperature spin correlation functions are concerned, this kind of property can be obtained from the hightemperature expansion. In a general context, the equivalence between the correlation function of equilibrium fluctuations and the law of relaxation was first explicitly postulated by Onsager ${ }^{28}$.

Assumption 2: On the long timescale, the relaxation of the slow quantities can be represented by Markovian first order rate equations, which can include only slow variables. These equations have to be linearised with respect to the small deviations from equilibrium.

Assumption 3: There are no slow variables that can affect $\langle X(t)\rangle$, other than $\langle X(t)\rangle$ itself.

Given the above assumptions, the only Markovian rate equation that can be written is

$$
\frac{d R}{d t}=-\gamma R
$$

where the new constant $\gamma$ is the decay rate defined by this equation. Assuming $R(0)=1$, the solution of Eq.(8) is $R(t)=\exp (-\gamma t)$.

It is, usually, at this point that the inapplicability of the Markovian description to short timescales is illustrated by pointing out that, on one hand, the Markovian approximation gives $\left.\frac{d R}{d t}\right|_{t=0}=-\gamma$, but, on the other hand, the time reversibility of the underlying dynamics guarantees that $\langle X(t) X(0)\rangle=\langle X(-t) X(0)\rangle$, which means $R(t)$ is an even function of time and, therefore, $\left.\frac{d R}{d t}\right|_{t=0}=0$.

The above contradiction does not invalidate the Markovian description, because, a priori, that description does not claim to provide the exact derivatives corresponding to the short-time fluctuations around the average long-time behavior. However, it is rarely emphasized that, in principle, there are two pictures of the short-time behavior of $R(t)$ that can be consistent with the long-time Markovian approximation: (a) smooth approach of $R(t)$ to the Markovian prediction or (b) persistent shorttime fluctuations of $R(t)$ around the average Markovian behavior. Both pictures are illustrated in Fig. 3.

Although, at first sight, it might seem that picture (b) better corresponds to the spirit of the Markovian approximation, the fact is: Whenever a theoretical at- 


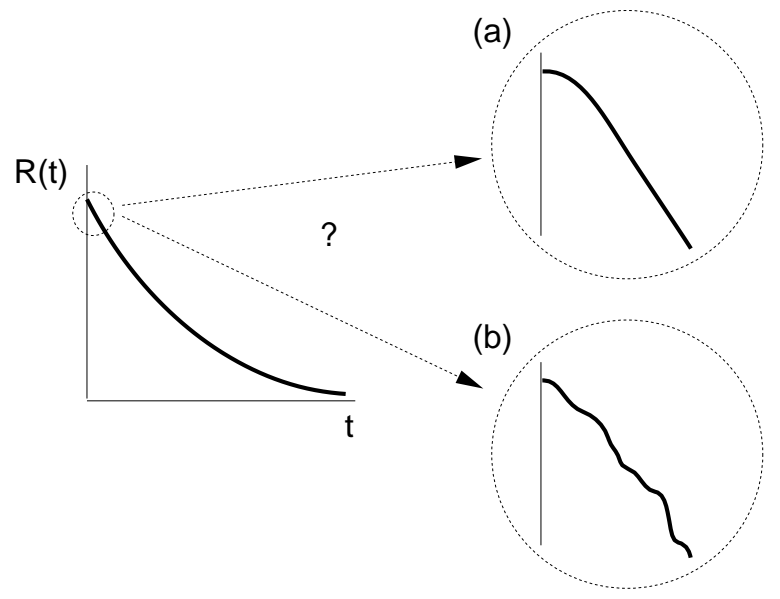

Fig. 3. This picture represents the correlation function $R(t)$ introduced in the text and, by magnifying the short timescale, shows two short-scale pictures compatible with the long-time Markovian description, namely: (a) smooth approach to the Markovian behavior with no further fluctuations and (b) persistent fluctuations around the average Markovian behavior. It is argued in the text that picture (a) is the one that corresponds to the reality.

tempt is made to embrace both the short- and the long-time behavior of $R(t)$, it results in picture (a). Moreover, we are not aware of any experiment or numerical calculation reliably showing the persistent short-time fluctuations of a correlation function around the average long-time behavior, provided that behavior is correctly predicted from a Markovian description.

Now we illustrate the assertion made in the previous paragraph by showing that the assumption of smooth approach to Markovian behavior is the only one that is necessary in order to obtain the Green-Kubo formula ${ }^{16,29}$ for the calculation of the constant $\gamma$ entering Eq.(8).

The smooth approach to Markovian behavior shown in Fig. 3(a) implies that the deviation of $R(t)$ from the Markovian prediction occurs only around $t=0$, and then the exact first time derivative of $R(t)$ approaches the value of $(-\gamma)$ within a short time interval, over which the value of $R(t)$ itself can change only insignificantly, and, after that, $R(t)$ follows the Markovian solution without any fluctuations. Within the same time interval, the second time derivative of $R(t)$ should change dramatically from a certain value characteristic of the short timescale to the value complying with the smooth and slow Markovian behavior.

The above description implies the following recipe for the calculation of relaxation rate $\gamma$ in Eq.(8): (i) Write the exact expression for the second derivative of $R(t)$ (ii) Perform the time integration of that expression until the value of the integral stops changing significantly on the fast timescale of the problem. The saturation value of that integral becomes the first derivative with which the correlation func- 
tion enters the Markovian regime. This value should be equated to $(-\gamma)$. In order to present the result in a familiar form, we note that $\langle\ddot{X}(t) X(0)\rangle$ (the exact expression for the second time derivative of $R(t))$ can be transformed ${ }^{\mathrm{a}}$ to $-\langle\dot{X}(t) \dot{X}(0)\rangle$. Therefore,

$$
\gamma=\int_{0}^{\infty}\langle\dot{X}(t) \dot{X}(0)\rangle d t
$$

The infinite upper limit of this integral should be understood in an approximate sense - it implies an upper cutoff, which is much longer than the characteristic time of the fast environment but much shorter than $1 / \gamma$. In the context of statistical physics, Eq.(9) is best recognizable as the Green-Kubo formula, though its resemblance with the "golden rule" of second order perturbation theory is not a coincidence.

It is actually easy to find an ansatz of the form $R(t)=\exp (-a(t))$, where the function $a(t)$ quickly approaches $\gamma t$, but, at the same time, $\left.\frac{d R}{d t}\right|_{t=0}=0$, and initially, the second time derivative of $R(t)$ follows $-\langle\dot{X}(t) \dot{X}(0)\rangle$. Such an ansatz is given by the formula:

$$
R(t)=\exp \left[-\int_{0}^{t}\left(t-t^{\prime}\right)\left\langle\dot{X}\left(t^{\prime}\right) \dot{X}(0)\right\rangle d t^{\prime}\right] .
$$

In the context of the exchange narrowing problem, this formula was first obtained by Anderson and Weiss ${ }^{23}$ on the basis of a Gaussian random noise model.

Fast decaying correlation functions like $\langle\dot{X}(t) \dot{X}(0)\rangle$ are usually difficult to compute, but they can be either reasonably approximated or, sometimes, extracted from experiment. Extensive experience with the Green-Kubo formula leaves no doubt that it is a reliable quantitative recipe provided the long-time Markovian assumptions are adequate. It is, however, clearly seen from the above discussion that, if the assumption of smooth approach to the Markovian behavior is incorrect, then the Green-Kubo formula should be abandoned.

\subsubsection{General argument}

Now we present a general intuitive argument that indeed, as the above discussion suggests, there should be no persistent short-time fluctuations of the correlation

aThe possibility of such a transformation is the consequence of the fact that the origin of the time axis can be arbitrarily shifted by some value $t_{0}$ without changing the correlation function of the equilibrium fluctuations. The transformation proceeds as follows:

$$
\begin{gathered}
\left\langle\frac{d^{2} X(t)}{d t^{2}} X(0)\right\rangle=\left\langle\frac{d^{2} X\left(t+t_{0}\right)}{d t^{2}} X\left(t_{0}\right)\right\rangle \\
=\left\langle\frac{d^{2} X\left(t+t_{0}\right)}{d t_{0}^{2}} X\left(t_{0}\right)\right\rangle \\
=-\left\langle\frac{d X\left(t+t_{0}\right)}{d t_{0}} \frac{d X\left(t_{0}\right)}{d t_{0}}\right\rangle+\frac{d}{d t_{0}}\left\langle\frac{d X\left(t+t_{0}\right)}{d t_{0}} X\left(t_{0}\right)\right\rangle,
\end{gathered}
$$

In equilibrium, the second term obtained above must be equal to zero, and the first term is obviously equal to $-\langle\dot{X}(t) \dot{X}(0)\rangle$. 
function $R(t)$ around the exponential Markovian behavior.

Let us return to Assumption 1 made in Section 3.3.1, which postulated that $R(t)$ can be treated as a relaxation function. A subtlety associated with that assumption is that, on the one hand, it is given in terms of the quantity $\langle X(t)\rangle_{\rho_{R}(0)}$ requiring the averaging over the ensemble of the sample evolutions of the entire system, but, on the other hand, the same quantity is equally expected to represent just one sample evolution of the macroscopic system in the sense of a real experiment, in which the initial conditions are randomly set in compliance with the initial probability distribution $\rho_{R}(0)$, and then the value of $X(t)$ is measured. This can only be true, if $X(t)$ is - or can be considered as - either the sum or the average of infinitely many equivalent contributions from statistically independent parts of the system. Such a condition is, normally, fulfilled for any quantity accessible by a macroscopic measurement.

In the following, we assume that the variable $X$ is decomposable as stated above, i.e.

$$
X(t)=\sum_{m} \tilde{x}_{m}(t)
$$

where $\tilde{x}_{m}(t)$ denotes one of many equivalent contributions to $X(t)$. Therefore, the correlation function $R(t)$ can be rewritten as

$$
R(t) \simeq\left\langle\tilde{x}_{m}(t)\right\rangle_{\rho_{R}(0), m}
$$

where the additional average is taken over all trajectories $\tilde{x}_{m}(t)$ during the same sample evolution of the entire system.

In Section 3.3.1, the variable $X$ was assumed to be the only slow variable characterizing the large system - therefore, the dynamic evolution of the variables $\tilde{x}_{m}$ is supposed to exhibit fast fluctuations representative of the short (ballistic) timescale of the problem.

Considering the ensemble averaged quantity $\left\langle\tilde{x}_{m}(t)\right\rangle_{\rho_{R}(0), m}$, it is important to realize that any violent short-time event experienced by one trajectory $\tilde{x}_{m}(t)$ can be experienced simultaneously by many other trajectories. Moreover, another set of trajectories $\tilde{x}_{m}(t)$ can also go through an identical short-time event, shifted in time by an interval much shorter or much longer than the scale of this short-time event itself (see Fig. 4 for the illustration). In other words, any given short-time event can start at any given moment of time. Therefore, in order for the short timescale to be pronounced after averaging over all trajectories, it is necessary that not only the short-time events were present in each trajectory, but also the probability of those events must fluctuate on the same short timescale.

The short-time fluctuations around the Markovian behavior of ensemble averaged quantities are absent, because the probability of a given short-time event is determined by a very large number of random contributions from uncorrelated trajectories. Being an extremely well averaged quantity, the above probability does not fluctuate. Even if the fluctuation of the probability of a specific short-time event 


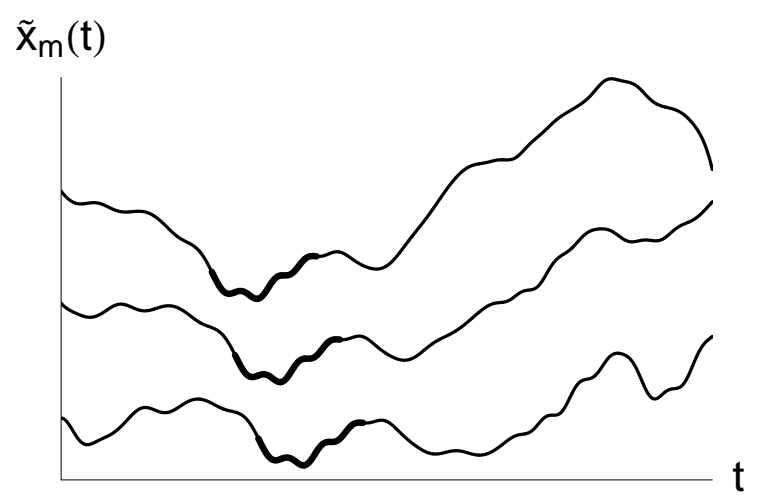

Fig. 4. This picture illustrates the argument made in the text that identical short-time pieces can appear in different trajectories of variable $\tilde{x}_{m}$. The three trajectories shown in this picture have one identical piece - the thicker part of each trajectory line. These trajectories have been displaced along the vertical axis to make them distinguishable. In this picture, the identical pieces are shifted in time by an interval which is shorter than the time length of each piece. In general, the time shift between the identical pieces can be both very short and very long.

accidentally occurs, then there are many different short-time events to be averaged over, and it is very unlikely, that the probability fluctuations for all of them happen at the same time and affect the average in the same direction.

To make the above discussion complete, one would have to answer the question: What is the difference between the initial probability distribution $\rho_{R}(0)$ and the probability distributions at later times that explains why the short-time deviation of $R(t)$ from the slow Markovian behavior occurs only around $t=0$ ? This question will be addressed in Sections 3.3.4 and 3.3.5 in a more general context.

\subsubsection{Conjecture I}

The preceding discussion motivated the postulate that whenever a kind of Markovian description is assumed to be adequate for long space- and timescales, the functional form of the correlation functions obtained in the framework of such a description also applies to the short timescale, with the only exception being an initial time interval of the order of the mean free time. As far as the corresponding relaxation process is concerned, then it is difficult to embrace that postulate without concluding that, after a certain time from the beginning of the nonequilibrium evolution, not only the resulting Markovian functional form but the Markovian rate equations themselves apply to the short timescales, and, if necessary, the spatial coarse-graining for the Markovian description can also be chosen very fine.

Making one step from the above seemingly formal observation, we now introduce a stronger statement:

Conjecture I: We consider a many-body system relaxing to equilibrium in the linear response regime and conjecture that in this process, after the memory of the 
initial probability distribution is lost, a kind of randomness subsequently propagates to very short space and time scales. This randomness is such that, considering the nonequilibrium behavior of the ensemble averaged quantities, it is appropriate to invoke a Brownian-like Markovian description, which is consistent with the longtime Markovian assumptions, but, at the same time, based on a very fine coarsegraining with the scale much smaller than the scale of the ballistic behavior of the particles composing the large system. (The precise meaning of the "Brownian-like Markovian description" will be clarified in Section 3.4 in a system-specific context.)

Two closely related aspects make the above conjecture different from the previous treatment: First, it does not require the quantity of interest to evolve much slower than a typical microscopic variable. Second, it does not call for the longtime Markovian description to be developed first and then extended to the short timescales - it only requires the long-time Markovian assumptions to be consistent with the resulting description.

The last circumstance makes Conjecture I complementary to the long-time assumption of chaotic mixing for the classical spin trajectories on a sphere (Section 3.2). As we have mentioned in Section 3.2, it is impossible to construct a self-contained long-time description on the spherical surface because of the physical absence of distances much greater than the mean free path. (The mean free path in our spin problem is of the order of the radius of the sphere). However, if the spherical surface is coarse-grained in very fine elements, then, locally, those elements can be considered flat. It is, therefore, natural to assume, that the random properties on the very fine scale in the case of random motion on the spherical surface are not qualitatively different from the case of Brownian motion on the infinite plane. Therefore, the rate equations similar to those used in Section 3.3.6 can be introduced on the spherical surface. An analogous situation will characterize the quantum case in Section 3.5.

\subsubsection{Local phase space picture for hyperbolic-like systems}

In this part, we introduce a complementary line of arguments involving the hypothesis that the many-body system addressed by Conjecture $I$ is a chaotic hyperbolic system ${ }^{16}$. The connection between Conjecture $I$ and the hypothesis of hyperbolicity is not an obvious one. We believe that hyperbolicity is not a necessary condition for Conjecture I, and we do not even speculate whether the hyperbolicity is sufficient to prove Conjecture I. In fact, only a very minimal consequence of hyperbolicity, the expansion-contraction picture, will be used in our arguments, and only in conjugation with "physical" (albeit mathematically uncontrollable) assumptions. Given all the above reservations, the origin and the limits of Conjecture I should, nevertheless, become more transparent when discussed in the context of hyperbolic systems.

The following discussion deals with an abstract many-body Hamiltonian system, about which it is no longer assumed that it exhibits separation between slow and fast motions. For consistency of language, the Hamiltonian system in question is 
assumed to be very large but finite. As a prototype of such a system, we keep in mind the lattice of interacting classical spins introduced in Section 1.1.

In the many-body phase space of a Hamiltonian system, the phase volume is conserved under dynamical evolution. When the whole system shows chaotic mixing, it means that any continuous set of points initially occupying a small part of the phase space would eventually be distributed over the whole phase space - subject to the energy conservation constraint. (This property is consistent with the mixing we assumed in Section 3.2 for the individual particle trajectories but somewhat more restrictive.) A volume-preserving continuous procedure of spreading the above set of points is to expand a part of this set in certain (unstable) directions and contract it in other (stable) directions, keeping the volume unchanged. At a given point of the phase space, the stable and unstable directions can be obtained by the linearization of the equations of motion in the vicinity of that point. Assuming that the dynamical evolution ensures such an expansion-contraction (hyperbolic) property for most points and most directions in the phase space, the initially compact small volume will be spread over the phase space as a continuous random pattern of very thin cells. The longer is the time allowed for this process, the smaller is the ultimate transverse size of the thin cells. This transverse size corresponds to the contraction directions of the phase space. It characterizes the scale of dynamically developed randomness and thus underlies the propagation of the Markovian assumptions to very short scales.

At the same time, if a smooth not-too-fast varying probability distribution is assigned within some initial small volume, such a distribution will be stretched over much larger phase space, and, therefore it will become nearly flat along the longitudinal (expansion) directions of the above cells. This extreme smoothness of the random pattern along the expansion directions makes the integrated average over large subvolumes behave in the same way as the average over much smaller subvolumes, and, therefore, allows the Brownian-like description for the long space and time intervals to be extended to much shorter intervals.

The expansion-contraction picture is visualized in Fig. $5^{33}$. Although the probability distributions presented in that figure do not exhibit chaotic patterns, they are, nevertheless, sufficient to illustrate the qualitative difference between the factorized distribution (Fig. 5(a)) and the distributions emerging at later stages of the expansion-contraction process (Fig. 5(d)). That illustration, however, requires the following explanation.

Let us assume that a fine coarse-graining is introduced in Fig. 5 in the space of variables $\left(X_{1}, X_{2}\right)$, and then consider two coarse-grained elements indicated in that figure. These two elements are displaced from each other along the expansion direction. Initially (Fig. 5(a)), the averaging over each of them would give two probabilities substantially different from each other. At later stages of the expansioncontraction process (Fig. 5(d)), the two coarse-grained elements become connected via the cells of extremely smooth probability distribution, and, therefore, the aver- 
(a)

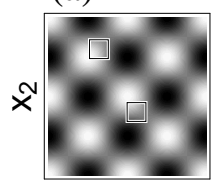

$\mathrm{X}_{1}$ (b)

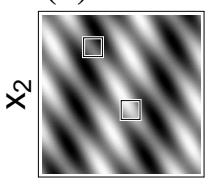

$\mathrm{X}_{1}$ (c)

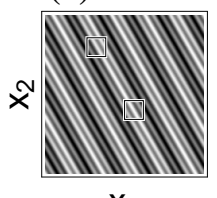

$\mathrm{X}_{1}$ (d)

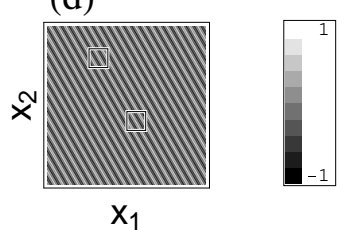

Fig. 5. Expansion-contraction picture - an oversimplified illustration to the arguments given in Section 3.3.4. Coordinates $X_{1}$ and $X_{2}$ represent a full many-body phase space. The interval shown along each axis is $(-2 \pi, 2 \pi)$. The coloring scheme (displayed in the rightmost frame) encodes function $f\left(X_{1}, X_{2}\right)$ proportional to a weak deviation from an equilibrium probability distribution. In figure (a), $f\left(X_{1}, X_{2}\right)=\cos X_{1} \cos X_{2}$ - it is an example of a factorized distribution. In figures (b-d), the distribution function from figure (a) is expanded by factor $k$ along the direction $(-\cos (\pi / 3), \sin (\pi / 3))$, and contracted by the same factor along the perpendicular direction $(\sin (\pi / 3), \cos (\pi / 3))$. The values of $k$ are the following: (b) $k=2$; (c) $k=8$; (d) $k=32$. Two small squares within each of the plots represent two sample coarse-grained elements discussed in the text.

aging over these elements would produce nearly identical results.

It might appear from Fig. 5 that the generic situation is the one, where two coarse-grained elements are displaced not along the expansion direction but along an arbitrary one. In that case, the two elements will not be connected via cells of smooth distribution.

The above objection, however, is based on an artifact of two-dimensional illustrations. A detail, which is difficult to represent graphically, is that the phase space of a system with a very large number of particles is many-dimensional, while, normally, the coarse-graining is applied to a few-dimensional subspace of that space (e.g. the subspace of one-particle coordinates). When a many-dimensional probability distribution exhibits an expansion-contraction pattern, the crucial difference of that pattern from the one presented in Fig. 5 is that the number of expansion directions is supposed to be much greater then the dimensionality of the coarse-grained subspace of interest. (In Fig. 5, the number of expansion directions is one, and the dimensionality of the coarse-grained space is two.) If a many-dimensional cell of smooth distribution has a projection on a given a few-dimensional coarse-grained element, then such a projection is overwhelmingly likely to be smoothly extended to all coarse-grained elements surrounding the given one.

We can now explain why Conjecture I distinguishes the initial and the long-time behavior of the correlation functions.

From the viewpoint of the expansion-contraction picture, the small parameter implicitly underlying Conjecture $I$ is the ratio of the characteristic scales of the many-body probability distribution along the contraction and expansion directions. 
Such a small parameter develops dynamically — it is not present in the initial probability distributions of a typical nonequilibrium problem.

As an example, let us consider our nonequilibrium problem for classical spins, in which case the initial probability distribution $\rho(0)$ is given by Eq.(4). According to Eq.(4), the probability of initial polarization of each spin is completely independent of the polarizations of its neighbors, which means that $\rho(0)$ can be factorized in terms of the distributions describing each spin separately. In essence, this factorization implies that a single scale (that of the initial one-spin distribution) characterizes the many-spin distribution along all directions of the phase space. That scale corresponds to the radius of the sphere on which the one-spin distribution is defined. (The explicit form of this distribution will be given in Section 3.4.) Thus, as always happens with well-defined initial conditions of many-body problems, the initial distribution (4) is not complex enough to discriminate between the contraction and expansion directions intrinsic for the many-body phase space of a given dynamical problem. Therefore, some time $(\sim \tau)$ is required before the chaotic pattern possessing the necessary small parameter is established (see Fig. 5).

\subsubsection{General scope of Conjecture I}

Returning to the argument given in Section 3.3.2, it is important in general, - and for the treatment of the quantum case in particular - that the argument is not limited to the case when the trajectories in question describe a subsystem within a much larger chaotic Hamiltonian system. In general, this argument applies when a subsystem is subjected to the influence of an external environment which follows continuous dynamics and has a continuous distribution. As long as the long-time mixing assumption is satisfied, the argument does not exclude a non-chaotic or non-Hamiltonian environment, and neither does Conjecture $I$.

In the case of a non-chaotic or non-Hamiltonian environment, the extreme stretching of the subsystem-plus-environment probability distribution (similar to the one described in Section 3.3.4) should also underly Conjecture I, though, in such a case, it is more difficult to visualize the small parameter. Nevertheless, it is clear that Conjecture $I$ becomes applicable after the probability distribution develops the complexity intrinsic to a given dynamics.

\subsubsection{One-dimensional Brownian motion}

In order to facilitate the Brownian-like description for classical spins in Section 3.4, here we describe the standard one-dimensional diffusion of Brownian particles in a fashion similar to the treatment of the exponential decay in Section 3.3.1. Instead of one slow variable considered in Section 3.3.1, the treatment of diffusion requires a continuum of slow variables.

In the problem of Brownian motion ${ }^{3029}$, the Markovian timescale should be associated with a slow change in the position coordinate $x(t)$ of a Brownian particle. 
The drift of Brownian particles is, usually, characterized by the correlation function

$$
B(t)=\left\langle x^{2}\right\rangle-\langle x(t) x(0)\rangle \text {. }
$$

We consider the problem as if many Brownian particles move simultaneously, and the average is taken over all of them.

In order to proceed with the Markovian description, it is necessary to introduce spatial coarse-graining with the size $\Delta_{x}$ of the coarse-grained subvolumes (linear intervals in this case). This size is much greater than the mean free path of the Brownian particles. The slowly changing particle populations in each subvolume become the primary variables of the Markovian description. It is then assumed that the Markovian equations should only describe the direct exchange of particles between adjacent subvolumes, which gives

$$
\frac{\partial f\left(t, x_{(i)}\right)}{\partial t}=W \sum_{j}\left(f\left(t, x_{(j)}\right)-f\left(t, x_{(i)}\right)\right),
$$

where $f\left(t, x_{(i)}\right)$ is the nonequilibrium fraction of the particle population in subvolume number $i$; the sum over $j$ includes only the subvolumes adjacent to the $i$ th subvolume; and $W$ is the rate of transitions across the boundary of two adjacent subvolumes. There are two additional assumptions behind Eq.(14). Namely: (a) every Brownian particle is always affected by the same equilibrated environment; and (b) the transitions in both directions $((i) \rightarrow(j)$ and $(j) \rightarrow(i))$ are equivalent. The continuum limit of the description represented by Eq.(14) yields the standard diffusion equation:

$$
\frac{\partial f(t, x)}{\partial t}=D \frac{\partial^{2} f(t, x)}{\partial x^{2}}
$$

where $D$ is the diffusion coefficient equal to $W \Delta_{x}^{2}$.

The solution of Eq.(15) allows $B(t)$ to be calculated, which gives

$$
B(t)=D t
$$

According to the general recipe preceding Eq.(9), the Green-Kubo expression for the diffusion coefficient can be obtained by equating the first time derivative of the Markovian result for $B(t)$ to the saturation value of the integral of the exact second time derivative of $B(t)$. After a manipulation, which is similar to the one as a footnote in Section 3.3.1, the second derivative of $B(t)$ can be presented as $\langle v(t) v(0)\rangle$, where $v(t)=\frac{d x(t)}{d t}$. Therefore, the expression for the diffusion coefficient becomes

$$
D=\int_{0}^{\infty}\langle v(t) v(0)\rangle d t
$$

Thus we have again illustrated the general statement that the assumption of the smooth approach to the Markovian behavior is sufficient in order to obtain the standard Green-Kubo results for the parameters describing the Markovian approximation. 


\subsection{Classical spins - continued}

Conjecture I can now be applied to the system of classical spins in the following way.

Let us assume that, on the spherical surface corresponding to the $n$th spin, we trace a large number of sample trajectories of that spin. Different sample trajectories of the $n$th spin correspond to different realizations of the dynamical evolution of the entire system, and the initial conditions for each such a realization are chosen in accordance with the initial probability distribution (4). We thus have a large number of sample points moving simultaneously on the spherical surface.

Now let us coarse-grain the spherical surface in sufficiently small elements. The greater is the target accuracy of the calculation, the finer is the coarse-graining. The large number of sample trajectories should guarantee that, at any moment of time, there are enough sample points in each coarse-grained subvolume (surface element, in this case) to give an accurate representation of the probability to find a point in this subvolume.

The probability distribution of the sample points on the $n$th sphere is initially close and expected to remain close to the uniform distribution corresponding to the infinite temperature equilibrium. We denote the small deviation from the uniform distribution as $f_{n}\left(t, x_{n}\right)$, where $x_{n}$ stands for two standard spherical angles $\theta_{n}$ and $\varphi_{n}$, chosen such that $S_{n}^{\mu}=S \cos \theta_{n}$, where $\mu$ is the same as the one entering Eq.(1). The expansion of $\rho(0)$ in powers of $\beta_{0}$ gives

$$
f_{n}\left(0, x_{n}\right) \simeq \beta_{0} \cos \left(\boldsymbol{q} \cdot \boldsymbol{r}_{n}\right) S \cos \theta_{n} .
$$

We now recall, that, in Section 3.2, we have already introduced the notion of the mean free path of a typical sample point on the above spherical surface.

When the coarse-grained scale is smaller than that mean free path, the influx and outflux events for the subvolumes along a given trajectory will be correlated in time. However, for a given subvolume, there should be many sample points entering and leaving it within any finite time interval. In equilibrium, the non-Markovian influx and outflux events should be perfectly balanced by the exact dynamics of the system. It is the slight imbalance between the influx and the outflux events that governs the nonequilibrium evolution in that subvolume. The property underlying Conjecture I can now be reformulated as follows: At the later stages of the ensemble's evolution, there are no important correlations between the uncompensated influx and outflux events for a given coarse-grained subvolume at the given and at the previous moments of time. The time interval between the two moments in question can be substantially shorter than the time required for a sample point to cross that subvolume.

In the long-time regime describable by Conjecture $I$, the population of sample points in a given subvolume becomes a "slow" variable. In Conjecture I, by postulating the applicability of Brownian-like description, we implied that the problem can now be treated as if the population of the sample points in each subvolume 
describes Brownian particles with mean free path much smaller than the size of that subvolume.

If the $n$th spin was in an equilibrium environment, then the description of Brownian motion presented in Section 3.3.6 could be adopted, and the continuum limit of such a description would lead to a diffusion equation similar to Eq.(15). However, in our case, the environment, which means spin distributions on the neighboring lattice sites, is slightly out of equilibrium.

At the level of rate equation (e.g. Eq.(14)), the leading order effect caused by the nonequilibrium environment is that the transition rate from one subvolume to another becomes slightly different from the transition rate in the opposite direction. In that case, the equilibrium fractions of particles in the two subvolumes respond to the disbalance of the transition rates and create the additional particle flux between those subvolumes. Below, we proceed directly to the continuum description, which takes into account the above effect.

In order to avoid unnecessary details, we adopt a schematic notation, which makes all quantities look one-dimensional, but implies the proper number of dimensions and the proper covariant form of the differential operations. In such a notation, the linear response formula for the probability flux on the $n$th site can be written as

$$
\begin{aligned}
j_{n}\left(t, x_{n}\right)= & -D_{n}\left(x_{n}\right) \frac{\partial f_{n}\left(t, x_{n}\right)}{\partial x_{n}} \\
& +\sum_{k} \int_{V_{x_{k}}} K_{n k}\left(x_{n}, x_{k}\right) f_{k}\left(t, x_{k}\right) d x_{k},
\end{aligned}
$$

where $D_{n}\left(x_{n}\right)$ is the diffusion coefficient (actually, tensor) corresponding to the standard diffusion in the equilibrium environment; $K_{n k}\left(x_{n}, x_{k}\right)$ is the kernel of the term representing the linear flux response on the $n$th site to the slight deviation from the equilibrium distribution on the $k$ th site (this response is analogous to Ohm's law); and $V_{x_{k}}$ stands for the entire space of variables characterizing the $k$ th site.

The probability density $f_{n}$ and the probability flux $j_{n}$ must also satisfy the continuity equation

$$
\frac{\partial f_{n}\left(t, x_{n}\right)}{\partial t}=-\operatorname{div}\left[j_{n}\left(t, x_{n}\right)\right]
$$

In general, pairs of equations $(19,20)$ should be written for each lattice site, and together they form a closed set of integro-differential equations.

Although introduced above as a natural development of the previous treatment, the integral term in Eq.(19) represents quite a dramatic step in the extension of the Brownian motion formalism beyond the limits of the conventional Markovian approximation. This term should absorb the seemingly intractable dynamical correlations between different spins. A simple picture behind that term is the following: The nonequilibrium probability of a certain orientation of the $k$ th spin creates a preferred direction of the local field by which the $k$ th spin affects the $n$th spin. In 
turn, the preferred direction of the local field means the preferred direction for the additional probability flux on the $n$th spherical surface.

In order to promote the Brownian-like description consistently, it is necessary to assume that the response of one slow variable (the population of a given subvolume) to the deviation of another slow variable from the equilibrium can be caused not only by the direct interaction between those variables but also by the indirect effects mediated by other spins. This means that there can exist a nonzero kernel $K_{n k}\left(x_{n}, x_{k}\right)$ coupling the $n$th and the $k$ th spins, even though those spins do not interact directly. Another kernel not to be neglected is $K_{n n}\left(x_{n}, x_{n}^{\prime}\right)$. In general, it is only reasonable to expect that, when the distance between the spins becomes much greater than the radius of interaction, the indirect effects become insignificant.

The evaluation of $D_{n}\left(x_{n}\right)$ and $K_{n k}\left(x_{n}, x_{k}\right)$ from the knowledge of the Hamiltonian (2) is not attempted in this work, and, in fact, we are not sure if such a task is achievable - the absence of a self-contained long-scale description on a sphere precludes us from applying a straightforward generalization of the GreenKubo recipe (17). Nevertheless, as we show below, it is possible to come to very definite conclusions about the time-dependences of the resulting solutions.

When the combined symmetry of the Hamiltonian and the initial conditions is such that two initial distributions $f_{n}\left(0, x_{n}\right)$ and $f_{k}\left(0, x_{k}\right)$ can be transformed into each other, then the dynamic evolution induced by the Hamiltonian retains that equivalence. Thus those two sites can be characterized by the same probability distribution also in the long-time diffusive regime.

The nonequilibrium problem corresponding to the calculation of the correlation function (1) can now be greatly simplified, because in this problem all probability distributions $f_{n}\left(t, x_{n}\right)$ are equivalent. This equivalence is related to the fact that the irreducible representations of the group of lattice translations have form $\exp \left(i \boldsymbol{q} \cdot \boldsymbol{r}_{n}\right)$ (cf. Eq. (18)).

The symmetry argument can be illustrated using the examples presented in Fig. 1(b). In that figure, all spins are explicitly equivalent in example (I). It is also quite obvious that the probability distributions corresponding to the alternating spin sites in example (II) are also equivalent. In the other two examples, one would have to imagine that the probability distribution of each spin is the real part of a complex-valued function which is the same for all spins up to the helically arranged phase.

It thus follows that, in our problem,

$$
\left.f_{n}\left(t, x_{n}\right)\right|_{x_{n}=x_{0}}=\cos \left(\boldsymbol{q} \cdot \boldsymbol{r}_{n}\right) f_{0}\left(t, x_{0}\right)
$$

Given the above relationship one can easily obtain from Eqs. $(19,20)$ that

$$
\frac{\partial f(t, x)}{\partial t}=\frac{\partial}{\partial x}\left(D(x) \frac{\partial f(t, x)}{\partial x}-\int_{V_{x^{\prime}}} K\left(x, x^{\prime}\right) f\left(t, x^{\prime}\right) d x^{\prime}\right),
$$


where $f(t, x) \equiv f_{0}\left(t, x_{0}\right)$, and

$$
K\left(x, x^{\prime}\right)=\sum_{k} \cos \left(\boldsymbol{q} \cdot \boldsymbol{r}_{k}\right) K_{0 k}\left(x, x^{\prime}\right) .
$$

Equation (22) represents correlated diffusion on a spherical surface. Like an ordinary diffusion equation, this equation has a set of solutions of the form $f_{(\lambda)}(t, x)=e^{-\lambda t} u_{\lambda}(x)$, where $\lambda$ is one of the eigenvalues of the integro-differential linear operator acting on $f(t, x)$ in the right-hand side of Eq.(22), and $u_{\lambda}(x)$ is the corresponding eigenfunction.

Unlike a typical diffusion problem, the eigenvalues of our problem can have both real and imaginary parts. This property originates from the fact that, in general, the kernel $K\left(x, x^{\prime}\right)$ has no symmetry with respect to interchange of the variables $x$ and $x^{\prime}$, and, as a result, the integro-differential operator in Eq.(22) is non-Hermitian.

The asymmetry of the kernel $K\left(x, x^{\prime}\right)$ can be traced back to the asymmetry of the kernels $K_{0 k}\left(x, x^{\prime}\right)$ entering Eq.(23). Although it is impossible to separate various factors influencing the kernels $K_{0 k}\left(x, x^{\prime}\right)$, it is, at least, clear that those kernels are strongly affected by the direct spin-spin interaction (2). That interaction is asymmetric in the sense that, in general, the $k$ th spin with orientation $x^{\prime}$ creates on the zeroth site a local field, which is different from the local field created on the $k$ th site by the zeroth spin with some arbitrary orientation $x$.

Since we deal with a finite volume (meaning spherical surface), the eigenfunctions $u_{\lambda}(x)$ necessarily form a discrete set and generate a corresponding discrete set of eigenvalues. (If the eigenvalues of our problem are complex, then only the real part of the solution should be taken.) We further assume that the equilibrium is stable, and, therefore, the underlying dynamics of the spin system guarantees that the real parts of all eigenvalues are non-negative.

In order to obtain $G(t)$ from the above description, one has to average the $\mu$ th polarization of the zeroth spin over the solution of Eq.(22). Therefore,

$$
\begin{aligned}
G(t) & \simeq \int_{\text {sphere }} \cos \theta f(t, \theta, \varphi) \sin \theta d \theta d \varphi \\
& =\int_{\text {sphere }} \cos \theta \sum_{\lambda} e^{-\lambda t} u_{\lambda}(\theta, \varphi) \sin \theta d \theta d \varphi
\end{aligned}
$$

where we returned to the spherical variables $\{\theta, \varphi\}$ introduced earlier (with index $n$ dropped). As a result, the generic long-time behavior of $G(t)$ is given by Eqs. $(5,6)$ - it is controlled by the eigenvalue that has the smallest real part among those, whose respective eigenfunctions $u_{\lambda}(\theta, \varphi)$ give nonzero contribution to the integral in Eq.(24).

The alternatives to the long-time behavior $(5,6)$, which we left out as "nongeneric", correspond to the following possibilities: (i) the system does not experience chaotic mixing sufficient to justify the diffusion description $(19,20)$; (ii) within the diffusion description, the expansion of $G(t)$ contains more than one eigenvalue with the smallest real part (complex conjugates do not count). 
In a typical case, the problem has only one characteristic timescale $\tau$ given by Eq.(3). Therefore, we expect the characteristic scales of both $D(x)$ and $K\left(x, x^{\prime}\right)$ to be given by the appropriate combination of $\tau$ and the radius of the sphere. With this, we can make the following simple estimate of the time, after which the exponential dependence (5) or (6) should become pronounced.

It presumably takes a time of the order of $\tau$ to reach the Markovian regime describable by Eq.(22). In the Markovian regime, both the slowest exponent $\xi$ entering Eqs. $(5,6)$, and the difference between $\xi$ and the second slowest exponent should be of the order of $1 / \tau$, implying that it will take another time of the order of $\tau$ before the contributions from the faster exponents become suppressed. Therefore, the behavior of $G(t)$ should approach asymptotic form $(5,6)$ after a time of the order of several $\tau$, i.e. sufficiently fast.

In the context of experimental or numerical verification of the long-time behavior $(5,6)$, the estimate just given should be complemented by reasonably good luck. For example, if the difference between the slowest exponent and the second slowest exponent equals one third of the slowest exponent, then the two exponents still compete over quite an extended time interval, and by the time the faster of those exponents becomes completely suppressed, the overall value of the correlation function becomes too small to be obtained from experiments or from numerical calculations.

\subsection{Quantum spins}

Generalization of the previous treatment to quantum spins requires one modification, namely, instead of trajectories of the tip of the classical spin on a sphere, we consider trajectories in the space of parameters describing the density matrix of a quantum spin.

As straightforward as the above approach might seem, it is not how Markovian assumptions are usually introduced in quantum problems. Usually, Markovian assumptions are based either on the classical limit of the quantum problem, or on classical models for the occupation numbers of the quantum states (e.g. Ising model). Both of those approaches are deficient because of the lack of invariance with respect to the transformations of the Hilbert space of the quantum problem. Sometimes (not in our case), such a deficiency appears to be unimportant, but it always makes the discussion of compatibility of the Markovian assumptions with the exact dynamics intractable even at the level of posing the problem. If the Markovian assumptions are to be discussed in a situation where their extreme implications are expected to be quantitatively adequate, it is crucially important to have those assumptions grounded on the well-defined notion of trajectories consistent with the symmetry of the Hilbert space.

A more recent trend is to relate the notion of chaos in quantum systems to the Wigner-Dyson statistics of energy levels. (See e.g. Refs. ${ }^{31,32}$.) In this work, however, 
the Wigner-Dyson statistics is neither assumed nor derived from another assumption. We can only remark that the spin $1 / 2 X X Z$ chains discussed in Section 2 do not exhibit the Wigner-Dyson statistics ${ }^{31}$ but show the long-time behavior (6).

Below, in order to be specific, we limit our treatment only to spins $1 / 2$. We also assume that the spin system is very large but finite, and, therefore, the number of states in the quantum basis is also finite.

The $2 \times 2$ density matrix of a given spin $1 / 2$ can always be obtained by averaging over the full many-body density matrix. Already an ensemble average, the one-spin density matrix is expected to show, not a random behavior, but, in our case, a rapid relaxation toward the $2 \times 2$ unit matrix corresponding to the infinite temperature equilibrium. Therefore, in order to identify the underlying chaotic trajectories, we have to trace back the quantum mechanical and statistical averaging.

We propose a description that treats different spins by using different basis sets of the many-body wave functions. Considering the $n$th spin, we adopt an interactionlike representation for the basis wave functions: First, at $t=0$, for the whole system excluding the $n$th spin, we choose a complete orthogonal set of wave functions $\left\{\Psi_{n \alpha}(0)\right\}$ enumerated by index $\alpha$. Each wave function $\Psi_{n \alpha}(0)$ is such that every spin has definite projection on the axis $\mu$ entering Eq.(4). (The spins are weakly polarized along that axis at $t=0$.) Allowing each of the above wave functions to evolve only under the action of the part of the Hamiltonian (2) not involving the $n$th spin, we obtain a time-dependent basis set $\left\{\Psi_{n \alpha}(t)\right\}$. Then, we choose time-independent basis wave functions for the $n$th spin as $\{|\uparrow\rangle,|\downarrow\rangle\}$ with the quantization axis along the same $\mu$-direction of the initial average polarization of this spin. Finally, the basis set for the whole system becomes $\left\{\left\{|\uparrow\rangle \Psi_{n \alpha}(t)\right\},\left\{|\downarrow\rangle \Psi_{n \alpha}(t)\right\}\right\}$.

According to the above recipe, the basis sets designed for the description of different spins are identical at $t=0$, but then each of those sets evolves differently. The advantages of this representation will become clear after the formal structure of our treatment is developed.

In the basis designed for the $n$th spin, the density matrix of that spin is just the average over $2 \times 2$ blocks of the density matrix of the whole system - each block corresponds to a fixed value of index $\alpha$. However, these blocks cannot yet be treated as elementary dynamical quantities, because the initial many-body density matrix (4) is, by itself, the result of statistical averaging, implying initial thermal contact with the environment.

Since, in our problem, there is no contact with the environment at later moments of time, we represent the evolution of the density matrix of the whole system as the average over the evolutions of many density matrices of "pure states". Pure states are the states of the isolated spin system - each describable by a wave function. The choice of the ensemble of pure states (to be specified later) is not unique, because, provided the averaging over the pure state density matrices at $t=0$ gives the density matrix (4), the result of the averaging at later moments of time must be independent of other details of this choice.

The time-dependent $2 \times 2$ density matrix of the $n$th spin can now be considered 
as an average over $2 \times 2$ time-dependent blocks, each originating from some pure state density matrix. If one of the pure state wave functions is represented by a set of coefficients $\left\{\left\{C_{\uparrow n \alpha}(t)\right\},\left\{C_{\downarrow n \alpha}(t)\right\}\right\}$ in the basis $\left\{\left\{|\uparrow\rangle \Psi_{n \alpha}(t)\right\},\left\{|\downarrow\rangle \Psi_{n \alpha}(t)\right\}\right\}$, then the elementary block participating in the averaging is

$$
\left(\begin{array}{cc}
C_{\uparrow n \alpha}(t) C_{\uparrow n \alpha}^{*}(t) & C_{\downarrow n \alpha}(t) C_{\uparrow n \alpha}^{*}(t) \\
C_{\uparrow n \alpha}(t) C_{\downarrow n \alpha}^{*}(t) & C_{\downarrow n \alpha}(t) C_{\downarrow n \alpha}^{*}(t)
\end{array}\right) .
$$

Each block of the form (25) can be described by three independent variables: $\left\{\left|C_{\uparrow n \alpha}\right|,\left|C_{\downarrow n \alpha}\right|, \Phi_{n \alpha}\right\}$, where $\Phi_{n \alpha}$ is the difference between the phases of $C_{\uparrow n \alpha}$ and $C_{\downarrow n \alpha}$. Therefore, the time evolution of each of those blocks can be mapped to a trajectory of the tip of a Bloch vector in the three-dimensional space corresponding to the above set of variables. The trajectories thus defined become the primary objects in the subsequent treatment. We shall call them "block trajectories".

In the following, when no distinction between $C_{\uparrow n \alpha}(t)$ and $C_{\downarrow n \alpha}(t)$ has to be made, we shall use the notation $C_{\uparrow(\downarrow) n \alpha}$. We shall also use variables $\left\{\left|C_{\uparrow n}\right|,\left|C_{\downarrow n}\right|\right.$, $\left.\Phi_{n}\right\}$ (the same as $\left\{\left|C_{\uparrow n \alpha}\right|,\left|C_{\downarrow n \alpha}\right|, \Phi_{n \alpha}\right\}$ but without index $\alpha$ ), whenever we discuss one block trajectory as a representative of the statistical properties of all trajectories with different $\alpha$. In particular, we introduce the probability distribution $P_{n}\left(t,\left|C_{\uparrow n}\right|,\left|C_{\downarrow n}\right|, \Phi_{n}\right)$, which implies averaging over the whole ensemble of the pure states and over all values of the index $\alpha$. This probability distribution will play the same role as the probability distribution on a sphere played earlier in the case of the classical spins.

We can now explain two advantages of the "interaction representation" basis $\left\{\left\{|\uparrow\rangle \Psi_{n \alpha}(t)\right\},\left\{|\downarrow\rangle \Psi_{n \alpha}(t)\right\}\right\}$.

The first advantage is that, in this representation, interactions which do not affect the $n$th spin directly also do not have a direct influence on the evolution of the block trajectories - those interactions are mostly absorbed by the time dependence of $\Psi_{n \alpha}(t)$. The time dependence of the coefficients $C_{\uparrow(\downarrow) n \alpha}(t)$ is directly controlled only by those terms in the Hamiltonian (2) that include the $n$th spin. If the wave functions $\left\{\left\{|\uparrow\rangle \Psi_{n \alpha}(0)\right\},\left\{|\downarrow\rangle \Psi_{n \alpha}(0)\right\}\right\}$ were used as a permanent basis set, then the interactions between two spins very distant from the $n$th spin would have immediate effect on the coefficients $C_{\uparrow(\downarrow) n \alpha}$, which does not affect the final average for the $n$th spin but introduces irrelevant fast timescales in the behavior of those coefficients.

The second advantage of the interaction-like representation is that each of the wave functions $\Psi_{n \alpha}(t)$ can be considered as representing a generic evolution of the environment of the $n$th spin, and, therefore, as far as the properties of that spin are concerned, it is sensible to use one probability distribution $P_{n}\left(t,\left|C_{\uparrow n}\right|,\left|C_{\downarrow n}\right|, \Phi_{n}\right)$ to describe all block trajectories corresponding to different values of the index $\alpha$.

Below we specify the initial probability distribution of the coefficients $C_{\uparrow(\downarrow) n \alpha}$ for the ensemble of many-body pure states. As we have already mentioned, this distribution is only constrained by the requirement that the average of the initial pure state density matrices is equal to the density matrix $\rho(0)$ given by Eq.(4). We 
impose one additional constraint necessary for the consistency of our treatment: Namely, we require that the initial probability distribution $P_{n}\left(0,\left|C_{\uparrow n}\right|,\left|C_{\downarrow n}\right|, \Phi_{n}\right)$ in the space of block trajectories should be such that it is only slightly different from the "equilibrium" probability distribution $P_{n}\left(\infty,\left|C_{\uparrow n}\right|,\left|C_{\downarrow n}\right|, \Phi_{n}\right)$.

Since

the object of our interest is the probability distribution $P_{n}\left(0,\left|C_{\uparrow n}\right|,\left|C_{\downarrow n}\right|, \Phi_{n}\right)$ rather than the full probability distribution of the pure states, the details characterizing the latter will be given only to the extent sufficient to define the former.

The fact that the initial density matrix (4) is diagonal in the "initial" basis $\left\{\left\{|\uparrow\rangle \Psi_{n \alpha}(0)\right\},\left\{|\downarrow\rangle \Psi_{n \alpha}(0)\right\}\right\}$ allows us to assign equal probability to all values of the complex phases of the coefficients $C_{\uparrow(\downarrow) n \alpha}$, which implies that the probability distribution with respect to $\Phi_{n \alpha}$ is uniform for each value of $\alpha$. Therefore,

$$
P_{n}\left(0,\left|C_{\uparrow n}\right|,\left|C_{\downarrow n}\right|, \Phi_{n}\right)=\frac{1}{2 \pi} p_{n}\left(\left|C_{\uparrow n}\right|,\left|C_{\downarrow n}\right|\right),
$$

where $p_{n}\left(\left|C_{\uparrow n}\right|,\left|C_{\downarrow n}\right|\right)$ is the distribution of $\left|C_{\uparrow(\downarrow) n}\right|$ defined by the above equation.

The set of absolute values $\left\{\left|C_{\uparrow(\downarrow) n \alpha}\right|\right\}$ for a sample pure state can be considered as a vector in a high-dimensional space. Since $\sum_{\uparrow(\downarrow) \alpha}\left|C_{\uparrow(\downarrow) n \alpha}\right|^{2}=1$, the tip of that vector is restricted to a "quadrant" of the high-dimensional hypersphere, where each point has a non-negative projection on each axis. We define the probability of sampling on this "spherical quadrant" to be uniform, with small corrections reflecting the weak polarizations of spins. We do not define those corrections explicitly, because, as we show below, there is a simple direct way to see what the resulting distribution $p_{n}\left(\left|C_{\uparrow n}\right|,\left|C_{\downarrow n}\right|\right)$ must be.

Let us first obtain $p_{n}\left(\left|C_{\uparrow n}\right|,\left|C_{\downarrow n}\right|\right)$ in the case when there are no small corrections, which corresponds to infinite temperature. We denote such a distribution as $p_{n \infty}$.

According to the above sampling procedure, the probability distribution $p_{n \infty}\left(\left|C_{\uparrow n}\right|,\left|C_{\downarrow n}\right|\right)$ is an average over identical probability distributions for each of the coefficients $C_{\uparrow(\downarrow) n \alpha}$. The probability for any of the coefficients $C_{\uparrow(\downarrow) n \alpha}$ to have a value in the interval between $y$ (an auxiliary variable) and $y+d y$ is proportional to the "area" of the thus-restricted hypersurface, which is given by $A_{\left(N_{\mathrm{b}}-1\right)}\left(\sqrt{1-y^{2}}\right) d y / \sqrt{1-y^{2}}$, where $N_{\mathrm{b}}$ is the number of the states in the quantum basis of the entire system, and $A_{N_{\mathrm{b}}-1}\left(\sqrt{1-y^{2}}\right)$ is the surface area of the $\left(N_{\mathrm{b}}-1\right)$-dimensional hypersphere of radius $\sqrt{1-y^{2}}$. Since $A_{\left(N_{\mathrm{b}}-1\right)}\left(\sqrt{1-y^{2}}\right) \simeq$ $\left(\sqrt{1-y^{2}}\right)^{\left(N_{\mathrm{b}}-2\right)}$, and $N_{\mathrm{b}}$ is supposed to be very large, the overall dependence of the probability distribution on $y$ should be well approximated by $\exp \left(-\frac{N_{\mathrm{b}}}{2} y^{2}\right)$. As a result,

$$
p_{n \infty}\left(\left|C_{\uparrow n}\right|,\left|C_{\downarrow n}\right|\right)=\frac{\pi}{2 N_{\mathrm{b}}} \exp \left[-\frac{N_{\mathrm{b}}}{2}\left(\left|C_{\uparrow n}\right|^{2}+\left|C_{\downarrow n}\right|^{2}\right)\right] .
$$

Predictably, the mean value of $\left\langle\left|C_{\uparrow(\downarrow) n}\right|^{2}\right\rangle$ corresponding to $p_{n \infty}\left(\left|C_{\uparrow n}\right|,\left|C_{\downarrow n}\right|\right)$ is equal to the inverse number of basis states. 
When the inverse temperature $\beta_{0}$ appearing in Eq.(4) is small the initial probability distribution for the $\left|C_{\uparrow n}\right|$ should correspond to infinite temperature for "spinsup" and be Gaussian, with mean value $\left\langle\left|C_{\uparrow n}\right|^{2}\right\rangle$ proportional to the probability $\mathcal{P}_{n \uparrow}$ of finding "spin-up," while the distribution of $\left|C_{\downarrow n}\right|$ should also be Gaussian but with a slightly smaller mean value $\left\langle\left|C_{\downarrow n}\right|^{2}\right\rangle$ proportional to the probability $\mathcal{P}_{n \downarrow}$ of finding "spin-down". Both $\mathcal{P}_{n \uparrow}$ and $\mathcal{P}_{n \downarrow}$ are very close to $\frac{1}{2}$. Thus, taking into account only the leading order corrections to Eq.(27), we obtain

$$
p_{n}\left(\left|C_{\uparrow n}\right|,\left|C_{\downarrow n}\right|\right)=\frac{\pi}{2 N_{\mathrm{b}}} \exp \left[-\frac{N_{\mathrm{b}}}{4}\left(\frac{\left|C_{\uparrow n}\right|^{2}}{\mathcal{P}_{n \uparrow}}+\frac{\left|C_{\downarrow n}\right|^{2}}{\mathcal{P}_{n \downarrow}}\right)\right] .
$$

We assume that in the course of the nonequilibrium evolution, the probability distribution $P_{n}\left(t,\left|C_{\uparrow n}\right|,\left|C_{\downarrow n}\right|, \Phi_{n}\right)$ will ultimately evolve to

$$
P_{n}\left(\infty,\left|C_{\uparrow n}\right|,\left|C_{\downarrow n}\right|, \Phi_{n}\right)=\frac{1}{2 \pi} p_{\infty}\left(\left|C_{\uparrow n}\right|,\left|C_{\downarrow n}\right|\right),
$$

where $p_{\infty}$ is given by Eq.(27).

Comparing the block trajectories in the quantum case with the trajectories of the spin vectors on a sphere in the classical case, three differences can be observed.

The first difference is that, unlike the trajectories of individual classical spins, the block trajectories introduced for the description of the $n$th spin describe the whole system, because the coefficients $C_{\uparrow(\downarrow) n \alpha}(t)$ are taken from the many-body wave function.

The second difference is closely related to the first one. Namely, unlike the case when one sample point representing a given spin moves on a spherical surface in the course of a sample evolution of the whole system, many sample points corresponding to different blocks of the sample pure state actually move simultaneously in the space $\left\{\left|C_{\uparrow n}\right|,\left|C_{\downarrow n}\right|, \Phi_{n}\right\}$. Moreover, those sample points interact with each other, because the corresponding blocks are coupled by the quantum Hamiltonian. In other words, if we consider a given block trajectory constructed for the $n$th spin and representing the evolution of a pair of coefficients $C_{\uparrow n \alpha}(t)$ and $C_{\downarrow n \alpha}(t)$, then the immediate environment of that trajectory is characterized not only by the probability distributions $P_{k}\left(\left|C_{\uparrow k}\right|,\left|C_{\downarrow k}\right|, \Phi_{k}\right)$ of the spins that interact with the $n$th spin, but also by the distribution $P_{n}\left(\left|C_{\uparrow n}\right|,\left|C_{\downarrow n}\right|, \Phi_{n}\right)$ itself.

Finally, the third difference is that, in comparison with the spherical surface, which is periodic in all directions and, therefore, finite, the space of variables $\left\{\left|C_{\uparrow n}\right|,\left|C_{\downarrow n}\right|, \Phi_{n}\right\}$ is periodically closed only in the direction of $\Phi_{n}$. The situation is less straightforward along the $\left|C_{\uparrow n}\right|$ and $\left|C_{\downarrow n}\right|$-directions: Both variables are bound by zero from below, and also there is a global normalization condition $\sum_{\uparrow(\downarrow) \alpha}\left|C_{\uparrow(\downarrow) n \alpha}\right|^{2}=1$, which imposes the upper bound $\left|C_{\uparrow(\downarrow) n}\right| \leq 1$. However, the real upper bound is not 1 but a much smaller value imposed by the statistical constraint; this could already be seen from the fact that, according to both the initial (28) and the final (27) probability distributions, the typical value of the variables $\left|C_{\uparrow(\downarrow) n}\right|$ is of the order of $1 / \sqrt{N_{\mathrm{b}}}$, where the number of the basis states $N_{\mathrm{b}}$ is exponentially greater than supposedly large number of spins in the system. 
If evolution of a pure state starts from the values of all variables $\left|C_{\uparrow(\downarrow) n \alpha}\right|$ of the order of $1 / \sqrt{N_{\mathrm{b}}}$, then it is improbable that any of those variables can ever become close to 1 . What is overwhelmingly probable is that any $\left|C_{\uparrow(\downarrow) n \alpha}(t)\right|$ will stay within an upper bound of the order of $1 / \sqrt{N_{\mathrm{b}}}$ during a time interval of any reasonable length.

Given all the above differences, the block trajectories still have two properties allowing us to treat them in very much the same way as in Sections 3.2 and 3.4 we treated the classical trajectories on the spherical surface. These two properties are: (a) the mean free time of the block trajectories is given by the same onespin interaction time $\tau$ defined by Eq.(3); and (b) the mean free path of the block trajectories is of the order of the size of the finite volume to which those trajectories are constrained, i.e. $\sim \pi$ along the $\Phi_{n}$-direction and $\sim 1 / \sqrt{N_{\mathrm{b}}}$ along the $\left|C_{\uparrow(\downarrow) n}\right|-$ directions.

The reason why the one-spin interaction time characterizes the block trajectories is that, as was already mentioned, in the "interaction representation" we chose earlier, the evolution of the coefficients $C_{\uparrow(\downarrow) n \alpha}(t)$ is directly controlled only by the interaction of the $n$th spin with its neighbors - without that interaction, the coefficients $C_{\uparrow(\downarrow) n \alpha}$ would be time-independent.

The mean free paths given above originate from a simple estimate of how much the value of the individual coefficient $C_{\uparrow(\downarrow) n \alpha}$ can change over an interval of the order of $\tau$ - subject to the condition that all coefficients dynamically coupled with a given one have absolute values of the order of $1 / \sqrt{N_{\mathrm{b}}}$ and arbitrary complex phases.

Now we make the hypothesis that the block trajectories exhibit the chaotic mixing property of the same kind as was assumed in Section 3.2 for the trajectories of classical spins. Namely, we assume that on the scale characterized by the mean free time $\tau$ : (i) each block trajectory loses the memory of the initial position; (ii) a set of all block trajectories starting from the initial positions within an arbitrarily small subvolume of the statistically constrained part of the space of variables $\left\{\left|C_{\uparrow n}\right|,\left|C_{\downarrow n}\right|, \Phi_{n}\right\}$ disperses over that part in a random manner; and (iii) the statistics of the trajectory patterns of this set becomes representative of the statistics of the whole ensemble of the block trajectories. In correspondence with the previous discussion, the term "statistically constrained" implies not too large values of $\left|C_{\uparrow(\downarrow) n \alpha}\right|$ in comparison with $1 / \sqrt{N_{\mathrm{b}}}$.

Most of the arguments we used in Section 3.3 in order to justify Conjecture I were given in a general form and apply directly to the quantum problem posed in terms of the block trajectories. The only exception is the phase space picture motivated by the properties of hyperbolic chaotic systems (Section 3.3.4). In principle, one could try to introduce a similar picture in the $2 N_{\mathrm{b}}$-dimensional space representing the absolute values and the phases of all coefficients $C_{\uparrow(\downarrow) n \alpha}$, but we do not pursue this line. Instead, we rely on the general statement made in Section 3.3.5 that Conjecture I only requires that the environment which governs the evolution 
of a given subsystem has a continuous dynamics and a continuous probability distribution in the space of its variables. The environment of a given block trajectory certainly satisfies the above condition. (See the preceeding discussion of the "second difference" between the classical spin trajectories and the block trajectories.)

Starting from this point, our entire treatment of the classical spin systems can be directly translated to the quantum case. Below, we discuss the quantum case only to the extent that allows us to establish a correspondence with the mathematical construction obtained in Section 3.4 for the classical case.

As in the classical case, we apply Conjecture $I$ to the ensemble of the block trajectories and thus justify Eqs. $(19,20)$, in which $x_{n}$ now represents the three variables $\left\{\left|C_{\uparrow n}\right|,\left|C_{\downarrow n}\right|, \Phi_{n}\right\}$; and

$$
f_{n}\left(t, x_{n}\right)=P_{n}\left(t, x_{n}\right)-P_{n}\left(\infty, x_{n}\right),
$$

where $P_{n}\left(\infty, x_{n}\right)$ is given by Eqs. $(29,27)$.

As we have already mentioned, the statistical constraint limits the evolution of the block trajectories to a finite volume. From the viewpoint of the diffusion description, that constraint implies that the diffusion coefficients and the integral kernels in Eq.(19) rapidly approach zero as the values of variables $\left|C_{\uparrow(\downarrow) n}\right|$ become greater than $1 / \sqrt{N_{\mathrm{b}}}$.

The solution of the set of Eqs. $(19,20)$ yields the distribution function $f_{0}\left(t, x_{0}\right)$, from which the correlation function of interest can be obtained as the average polarization of the zeroth spin, i.e.

$$
G(t) \simeq\left\langle\left|C_{\uparrow 0}\right|^{2}\right\rangle(t)-\left\langle\left|C_{\downarrow 0}\right|^{2}\right\rangle(t) .
$$

For any wave vector $\boldsymbol{q}$ corresponding to the spatial period commensurate with the lattice periodicity, the same symmetry argument as in the classical case guarantees that the diffusion problem $(19,20)$ corresponding to the calculation of the correlation function (1) can be reduced to Eq.(22).

Even though Eq.(22) now describes the block trajectories, the finite volume argument asserting the discreteness of the spectrum is still applicable, and so are the estimates given in Section 3.4. Thus, similarly to Eq.(24), Eq.(31) can be expanded in terms of the discrete set of exponential decays:

$$
\begin{aligned}
G(t) & \simeq \int_{\left|C_{\uparrow 0}\right|,\left|C_{\downarrow 0}\right|, \Phi_{0}}\left(\left|C_{\uparrow 0}\right|^{2}-\left|C_{\downarrow 0}\right|^{2}\right) f_{0}\left(t,\left|C_{\uparrow 0}\right|,\left|C_{\downarrow 0}\right|, \Phi_{0}\right) d\left|C_{\uparrow 0}\right| d\left|C_{\downarrow 0}\right| d \Phi_{0} \\
& =\int_{\left|C_{\uparrow 0}\right|,\left|C_{\downarrow 0}\right|, \Phi_{0}}\left(\left|C_{\uparrow 0}\right|^{2}-\left|C_{\downarrow 0}\right|^{2}\right) \sum_{\lambda} e^{-\lambda t} u_{\lambda}\left(t,\left|C_{\uparrow 0}\right|,\left|C_{\downarrow 0}\right|, \Phi_{0}\right) d\left|C_{\uparrow 0}\right| d\left|C_{\downarrow 0}\right| d \Phi_{0}
\end{aligned}
$$

Therefore, we come to the conclusion that the asymptotic long-time behavior of $G(t)$ has the functional form (5) or (6). Such a behavior should become pronounced after the time of the order of several $\tau$. 


\subsection{Summary}

To summarize what was explained by our theory, we now give the answers to the questions posed in the end of Section 1.3.

Why is the long-time regime universal?

In the many-dimensional space representing all dynamical variables of the problem, the long-time regime is characterized by a pattern of the probability distribution, which is intrinsic to a given dynamical system (e.g. expansion-contraction pattern) and has the property that, on one hand, it is extremely complex, but, on the other hand, it develops cells of an extremely smooth structure that spread over the entire many-dimensional space.

What makes the long-time regime different from the initial regime?

The initial probability distribution is factorized, and, therefore, its pattern is qualitatively different from the intrinsic one.

Why does the long-time decay have functional form $(5,6)$ ?

The functional form of the long-time decay is determined by the slowest eigenmode of the problem of correlated diffusion in a finite volume. The finiteness of the volume is the consequence of two features of our quantity of interest. Namely, (i) we deal with spins, which means the finite volume of the phase space per lattice site; and (ii) we consider $q$-dependent correlation functions (see below).

Why are Eqs. $(5,6)$ primarily relevant to the $q$-dependent correlation functions (1) and not to the pair correlation functions of the form $\left\langle S_{k}^{\mu}(t) S_{n}^{\mu}(0)\right\rangle$ ?

In order for the diffusion problem to be posed in a finite volume, it is necessary that, at $t=0$, there are no more than a finite number of non-equivalent probability distributions on different lattice sites. The initial probability distributions for the diffusion problem associated with a $q$-dependent correlation function are, actually, all equivalent - consequence of the fact that they correspond to the irreducible representations of the group of lattice translations. For the diffusion problem associated with a pair correlation function, the number of non-equivalent probability distributions is infinite. One can only establish the exponential ceiling for the long-time decay in such a problem. It can be done by expanding the pair correlation function in the infinite series of the $q$-dependent correlation functions. The ceiling would then be given the $q$-dependent correlation function having the slowest long-time decay.

What mechanism is responsible for the oscillations in Eq.(6)?

The resulting diffusion description is non-Hermitian, which can be linked to the asymmetry in the motion of two interacting spins. The eigenvalues of the nonHermitian diffusion problem can have both real and imaginary parts. If present, the imaginary part of the slowest eigenmode leads to the oscillations.

\section{Factors affecting the competition between the monotonic and the oscillatory regimes}

The analysis to be presented in this Section cannot prove by itself that the correlation functions (1) should have the long-time behavior either of form (5) or (6). 
However, once the functional form of that behavior is established, it is not difficult to formulate semi-empirical rules that would allow one to anticipate the outcome of the competition between the monotonic and the oscillatory regimes. Such an analysis is possible, because the trends towards the monotonic or the oscillatory regime can already be extracted from the initial behavior of the correlation functions, which is, otherwise, not describable by the functional forms (5) or (6).

To begin with, the long-time regime of the correlation functions (1) depends on the form of the Hamiltonian, the value of the wave vector and the choice of the spin component $\mu$ (i.e. $x, y$ or $z$ ). A change in any one of the above three conditions can modify the behavior of the correlation functions from monotonic to oscillatory and vice versa.

We shall exemplify our analysis by the correlation functions (1) involving the $x$-components of spins (i.e. $\mu \rightarrow x$ in Eq.(1)). This covers all examples presented in Fig. 2, with the only exception of Fig. 2(b)(II).

We identify the following factors as important for discriminating between the two regimes: (i) direct correlations; (ii) motional narrowing; (iii) indirect correlations; (iv) number of interacting neighbors; and, finally, (v) quantum-to-classical crossover. These factors are listed in the order of their relative importance as derived from our experience. This order applies to most but not to every situation.

The factor of direct correlations can be evaluated by comparing two quantities. The first of them is the second moment of $G(t)$ :

$$
M_{2} \equiv-\left.\frac{d^{2} G}{d t^{2}}\right|_{t=0}=\frac{1}{3} S(S+1) \sum_{n}\left[{J_{k n}^{y}}^{2}+{J_{k n}^{z}}^{2}-2 J_{k n}^{y} J_{k n}^{z} \cos \left(\boldsymbol{q} \cdot \boldsymbol{r}_{k n}\right)\right]
$$

and the second one is $M_{2 u}$ - the second moment of the one-spin autocorrelation function

$$
u(t)=\frac{\left\langle S_{k}^{x}(t) S_{k}^{x}(0)\right\rangle}{\left\langle S_{k}^{x}(0) S_{k}^{x}(0)\right\rangle}
$$

The value of $M_{2 u}$ is:

$$
M_{2 u} \equiv-\left.\frac{d^{2} u}{d t^{2}}\right|_{t=0}=\frac{1}{3} S(S+1) \sum_{n}\left[J_{k n}^{y}{ }^{2}+{J_{k n}^{z}}^{2}\right] .
$$

The inequality $M_{2}<M_{2 u}$ indicates that the correlation between the average polarizations of neighboring spins decreases the rate of the initial decay of $G(t)$ in comparison with $u(t)$. In this case, $G(t)$ tends to exhibit monotonic behavior. In the extreme case when $M_{2} \ll M_{2 u}$, the characteristic time of the decay of $G(t)$ is much longer than the mean free time $\tau$. This justifies the Markovian approximation which would predict simple exponential decay.

In the opposite case, when $M_{2}>M_{2 u}$, the direct correlations favor oscillatory decay. It is, however, impossible to propose a Hamiltonian of form (2) that would lead to $M_{2} \gg M_{2 u}$. The best one can get is $M_{2}=2 M_{2 u}$, which is the case for Fig.2(c)(I). 
The difference between $M_{2}$ and $M_{2 u}$ is due to the term $2 J_{k n}^{y} J_{k n}^{z} \cos \left(\boldsymbol{q} \cdot \boldsymbol{r}_{k n}\right)$ in Eq.(33). Therefore, the sign of this difference depends on the sign of $\cos \left(\boldsymbol{q} \cdot \boldsymbol{r}_{k n}\right)$ and on the relative sign of $J_{k n}^{y}$ and $J_{k n}^{z}$.

The dependence on the relative sign of $J_{k n}^{y}$ and $J_{k n}^{z}$ is, at first sight, difficult to grasp intuitively. The origin of that dependence, however, becomes obvious when Hamiltonian (2) is decomposed in the following way ${ }^{12}$ :

$$
\begin{aligned}
\mathcal{H}= & \sum_{k<n} J_{k n}^{x} S_{k}^{x} S_{n}^{x}+\frac{1}{4}\left(J_{k n}^{y}-J_{k n}^{z}\right)\left(S_{k}^{+} S_{n}^{+}+S_{k}^{-} S_{n}^{-}\right) \\
& +\frac{1}{4}\left(J_{k n}^{y}+J_{k n}^{z}\right)\left(S_{k}^{+} S_{n}^{-}+S_{k}^{-} S_{n}^{+}\right)
\end{aligned}
$$

where $S_{k}^{+}=S_{k}^{y}+i S_{k}^{z}$, and $S_{k}^{-}=S_{k}^{y}-i S_{k}^{z}$.

As far as the correlation functions of the $x$-components of spins are concerned, only the second and the third terms in the above decomposition are responsible for the direct correlation effect. We refer to these two terms as the "double-flip term" and the "flip-flop term" respectively. The correlation trends associated with them are precisely opposite. When $J_{k n}^{y}$ and $J_{k n}^{z}$ have the same sign, the flip-flop term, dominates. In this case, the $x$-components of two interacting spins tend to change in the opposite directions, which, in the extreme case of $J_{k n}^{y}=J_{k n}^{z}$, leads to the conservation of the $x$-component of the total spin. When $J_{k n}^{y}$ and $J_{k n}^{z}$ have opposite signs, the double-flip term dominates, and, as a result the $x$-components of two interacting spins tend to change simultaneously in the same direction.

Each of the two kinds of direct correlations can favor either the monotonic or the oscillatory long-time behavior - the correspondence here can be made definite only after the wave vector $\boldsymbol{q}$ is specified. For example, when $q=0$, the double-flip term always favors the oscillatory decay, and the flip-flop term always favors the monotonic decay.

The direct correlation factor alone would favor oscillatory decay in the examples shown in Figs. 2(a) and (b) and in Figs. 2(c)(I) and (III), and monotonic decay in Fig. 2(c)(II). It is thus highlighted by Fig. 2(c)(III) that there are other important factors to consider.

The second factor among those listed earlier is motional narrowing. In our definition, motional narrowing is associated with the fact that, in general, the lifetimes of local-fields generated by the Hamiltonian (2) are finite. (In other words, those local fields change in time.) Here, we are concerned only with the local field components contributing to $M_{2}$ (i.e. the $y$ - and the $z$-components).

The reference to "narrowing" originates from the limit of very short lifetimes. In that limit, a Markovian approximation applies and, as usual, justifies simple exponential decay. That decay is much slower than one would expect just from the knowledge of $M_{2}$.

According to the Hamiltonian (2), the local field $\boldsymbol{h}_{k}$ that affects the $k$ th spin is 
given by

$$
\boldsymbol{h}_{k}=\sum_{n}\left[J_{k n}^{x} S_{n}^{x} \hat{\boldsymbol{e}}_{x}+J_{k n}^{y} S_{n}^{y} \hat{\boldsymbol{e}}_{y}+J_{k n}^{z} S_{n}^{z} \hat{\boldsymbol{e}}_{z}\right]
$$

where $\hat{\boldsymbol{e}}_{x}, \hat{\boldsymbol{e}}_{y}$ and $\hat{\boldsymbol{e}}_{z}$ are the unit vectors in the respective directions.

The lifetime of the $y$-component of the local field can be estimated as $\left[S^{2} \sum_{n}\left(J_{k n}^{x}{ }^{2}+J_{k n}^{z}{ }^{2}\right)\right]^{-1 / 2}$, and the lifetime of the $z$-component as $\left[S^{2} \sum_{n}\left(J_{k n}^{x}{ }^{2}+J_{k n}^{y}{ }^{2}\right)\right]^{-1 / 2}$.

Motional narrowing does not affect the second moments of correlation functions (1), but it does affect the higher moments. The effect of motional narrowing is always to shift the balance towards monotonic decay. The shorter are the lifetimes of the local fields, the stronger is the above trend.

The best way to expose the influence of motional narrowing is to change $J_{x}$, because the corresponding term in the Hamiltonian does not affect either $M_{2}$ or $M_{2 u}$, i.e. it does not contribute to the direct correlation effect discussed earlier. At the same time, the presence of that term reduces the lifetimes of both the $y$ - and the $z$-components of the local-fields.

Let us consider the example shown in Fig.2(c)(III). In this case, the direct correlation effect is modest. It favors oscillatory behavior, which would be the case if $J_{k n}^{x}=0$. However, the value of $J_{k n}^{x}$ is sufficiently large. As a result, the motional narrowing ultimately outweighs the direct correlations, and the decay becomes monotonic.

The $J_{k n}^{x}$-term is also important for the example shown in Fig.2(c)(II). Although the direct correlations in this case already favor monotonic decay, that trend would be much weaker without the $J_{k n}^{x}$-term.

The third factor to be discussed here is indirect correlations. Under this name, we refer to the correlations between spins that do not interact directly. These correlations cannot be reflected in the values of the second moments $M_{2}$ and $M_{2 u}$, but, otherwise, they are very similar to direct correlations.

We cannot formulate a simple rule for the trend caused by indirect correlations. We can only assert that, in general, indirect correlations do not modify the effect of direct correlations qualitatively. However, one has to be aware that the presence of indirect correlations introduces a substantial uncertainty to the earlier analysis, when the transfer of the $x$-polarization between different spins is efficient (i.e. $\left.\left|J_{k n}^{y}\right| \approx\left|J_{k n}^{z}\right|\right)$, but the wave vector $\boldsymbol{q}$ is such that the effect of direct correlations (i.e. the difference between $M_{2}$ and $M_{2 u}$ ) is anomalously small. When such a coincidence occurs (see e.g. fig.1(c) in Ref. ${ }^{26}$ ), our experience indicates that the effect of indirect correlations can be anticipated by assuming, that there exists an additional interaction of flip-flop type between the next-nearest neighbors.

The rationale for such a prescription is the following. In the leading order, the indirect correlations between two spins are due to their direct interaction with one common neighbor. These correlations involve either two double-flips or two flip-flops, but in the both cases, the resulting effect is of flip-flop type. The subtlety here is 
that nearly the same mechanism is responsible for the back-reaction effect, when the polarization of a given spin is transferred to its neighbor and then transferred back. Empirically, however, the indirect correlations coming from the next-nearest neighbors appear to dominate over the back-reaction effect.

The fourth factor in our list is the number of interacting neighbors. From our experience ${ }^{26}$, a smaller number of interacting neighbors tends to favor the oscillatory behavior, though, typically, this effect is not very significant.

Everything in the preceding analysis equally applies to both classical and quantum spin systems. However, the substitution of classical spins by quantum ones (especially spins $1 / 2$ ) is, by itself, a factor (the fifth one in our list), which, to some extent, favors the oscillatory regime. This factor is most pronounced in the situation with a few interacting neighbors. It can certainly be linked to the difference between the solutions of two-spin problems for spins $1 / 2$ and for classical spins.

Now we would like to discuss the transition between the monotonic and the oscillatory long-time regimes. For that purpose, it is helpful to bring out one of the results of our treatment in Section 3, namely, that the long-time behavior of the correlation functions (1) is, actually, the sum of many exponential terms, some of which can have the monotonic form (5) and others can have the oscillatory form (6).

That notion sheds light on the way in which the transition from the monotonic to the oscillatory decay proceeds as parameters of the Hamiltonian change. The likely scenario (which is also supported by our numerical experience) is that, at the transition, the exponential decay constants of both the slowest oscillating term and the slowest monotonic term become equal to each other. Therefore, as the transition approaches, the long-time behavior of the correlation functions should be parameterized as the sum of two terms - one having form (5) and the other having form (6).

The above description implies that, when the previous analysis does not give an unambiguous prediction, a transitional situation should be suspected, which means that the correlation function can exhibit a superposition of the two regimes during an extended initial time interval. Such a competition is, for example, present in Fig.2(c)(III), and also in some of the free induction decays reported in Ref. ${ }^{21}$.

Finally, we would like to mention a useful rigorous result obtained in Ref. ${ }^{13}$ (Ch.6.3.7) on the basis of the so-called Widom's theorem. Namely, the inequality $M_{4} / M_{2}^{2}<1.5$, where $M_{4}$ is the fourth moment of $G(t)$, guarantees that $G(t)$ has at least one zero, which, in turn, strongly suggests (but not guarantees) that the long-time decay of $G(t)$ has oscillatory form.

\section{Conclusions}

In this work, the treatment of spin systems was centered around Conjecture I, which extended a Brownian-like formalism to non-Markovian space- and timescales. If true, that conjecture is important for non-equilibrium physics in general. Another result 
of general interest is a novel formalism developed for the quantum systems, in order to apply the above conjecture. A potentially fundamental issue associated with Conjecture $I$ is the absence of apparent limits beyond which the exact dynamics of systems with infinite number of particles invalidates that conjecture.

It should be emphasized, that although we presented Conjecture I as a natural extension of physical and mathematical experience, it was used to address the properties of fast microscopic relaxation, which are generally considered very difficult to access, and for which not much reliable experience exists. At present, our theory based on Conjecture I can make only one solid prediction for the fast relaxation, namely, that the functional form of the generic long-time behavior of the infinite temperature spin correlation functions (1) is given by Eqs. $(5,6)$. The underlying description, however, has a substantial explanatory power and also allows estimates to be made.

In a separate development, we have also presented a semi-empirical description of various factors that discriminate between the monotonic long-time regime and the oscillatory one.

Finally, we would like to mention that both the theoretical estimates and the empirical evidence show that the knowledge of the long-time behavior $(5,6)$ has a practical value, related to the fact that this behavior becomes pronounced before the correlation functions (1) decay to impractically small values.

\section{Acknowledgements}

The author is grateful to A. J. Leggett for numerous discussions, to C. M. Elliott and to R. Ramazashvili for helpful comments on the manuscript, and to K. Fabricius for sharing some of his unpublished numerical data. A large part of this work has been done during the author's stay at the University of Illinois at Urbana-Champaign, where it has been supported in part by the MacArthur Chair endowed by John D. and Catherine T. MacArthur Foundation at the University of Illinois and by the Drickamer Endowment Fund at the University of Illinois. This work has also been supported during the author's stay at Spinoza Institute, University of Utrecht, by the Foundation of Fundamental Research on Matter (FOM), which is sponsored by the Netherlands Organization for the Advancement of Pure Research (NWO).

\section{Appendix A. Asymptotic law of the long-time relaxation and the memory function approach}

In this Appendix, we discuss the derivation of the long-time behavior $(5,6)$ by Borckmans and Walgraef ${ }^{6,7}$. (See also Ref. ${ }^{13}$ ). We recall that, even though the technique used by these authors is more sophisticated than the memory function $\operatorname{approach}^{5,8}$, it describes the long-time behavior of the free induction decay by the 
memory function equation

$$
\frac{d G(t)}{d t}=-\int_{0}^{t} F\left(t^{\prime}\right) G\left(t-t^{\prime}\right) d t^{\prime}
$$

where $F(t)$ is the memory function. This equation can be solved by the method of the Laplace transform, which leads to the following expression:

$$
\tilde{G}(s)=\frac{G(0)}{s+\tilde{F}(s)},
$$

where $s$ is the variable of the Laplace transform, and $\tilde{G}(s)$ and $\tilde{F}(s)$ are the Laplace transforms of $G(t)$ and $F(t)$ respectively.

It is not difficult to see that, under certain conditions (e.g. when $F(t)$ is Gaussian), $\tilde{G}(S)$ has a discrete set of poles, and, therefore, the pole closest to the imaginary axis controls the long-time behavior of $G(t)$, which means that the asymptotic behavior must have the functional form (5) or (6).

In essence, the proof of Borckmans and Walgraef consisted of exploiting the above fact in conjunction with several arguments that the shape of $F(t)$ should not differ much from Gaussian.

However, a closer examination of the problem reveals the following detail: The long-time behavior of $G(t)$ depends crucially on the long-time behavior of the memory function $F(t)$. If the long-time decay of $F(t)$ is slower than exponential, then it is easy to verify by direct substitution in Eq.(A.1) that $G(t)$ cannot decay exponentially either. Technically, the issue boils down to the fact that, when the long-time decay of $F(t)$ is slower than exponential, the Laplace transform of $F(t)$ is likely to have a branch cut passing through $s=0$. Therefore, Eq.(A.2) only helps to reformulate the difficult problem of the long-time behavior of $G(t)$ in terms of an even less tractable problem of the long-time behavior of $F(t)$, for which the crude estimates of the overall functional shape are not sufficient.

\section{References}

1. The findings of the present paper have been first reported in eprint: B.V. Fine, condmat/9911230 (1999); and in B. V. Fine, Ph. D. thesis, University of Illinois at UrbanaChampaign, (2000), available at http://www.physics.uiuc.edu/Research/Publications/theses/copies/fine.ps

2. E. Balcar and S. W. Lovesey, Theory of Magnetic Neutron and Photon Scattering (Clarendon, Oxford, 1981).

3. I. J. Lowe and R. E. Norberg, Phys. Rev. 107, 46 (1957).

4. A. Abragam, Principles of Nuclear Magnetism (Clarendon Press, Oxford, 1961), Chap. IV.

5. J. A. Tjon, Phys. Rev. 143, 259 (1966).

6. P. Borckmans and D. Walgraef, Phys. Rev. 167, 282 (1968).

7. P. Borckmans and D. Walgraef, Phys. Rev. Lett. 21, 1516 (1968).

8. G. W. Parker and F. Lado, Phys. Rev. B 8, 3081 (1973).

9. M. Engelsberg and N.-C. Chao, Phys. Rev. B 12, 5043 (1975).

10. A. A. Lundin, Zh. Eksp. Teor. Fiz. 102, 352 (1992) [Sov. Phys. JETP 75, 187 (1992)]. 
11. J. Jensen, Phys. Rev. B 52, 9611 (1995).

12. B. V. Fine, Phys. Rev. Lett. 79, 4673 (1997).

13. B. Cowan, Nuclear Magnetic Resonance and Relaxation (Cambridge University Press, 1997)

14. R. N. Shakhmuratov, J. Phys. Condens. Matter 3, 8683 (1991).

15. D. Ruelle, Phys. Rev. Lett. 56, 405 (1986).

16. P. Gaspard, Chaos, scattering and statistical mechanics (Cambridge University Press, Cambridge, 1998).

17. T. Prosen, J. Phys. A:Math. Gen. 35, L737 (2002).

18. T. Prosen, Physica D, in press (2003).

19. M. Engelsberg and I. J. Lowe, Phys. Rev. B 10, 822 (1974).

20. K. Fabricius, U. Löw, J. Stolze, Phys. Rev. B 55, 5833 (1997).

21. D. S. Metzger and J. R. Gaines, Phys. Rev. 147, 644 (1966).

22. K. Fabricius and B. M. McCoy, Phys Rev B 57, 8340 (1998).

23. P. W. Anderson, P. R. Weiss, Rev. Mod. Phys. 25, 269 (1953).

24. J. E. Guley, D. Hone, D. J. Scalapino, B. G. Silbernagel, Phys. Rev. B 1, 1020 (1970).

25. J. E. Guley, B. G. Silbernagel, V. Jaccarino, J. Appl. Phys. 40, 1318, (1969).

26. B. V. Fine, J. Stat. Phys. 112, 319(2003).

27. N. S. Krylov, Works on the Foundations of Statistical Physics, (Princeton University Press, Princeton, 1979)

28. L. Onsager, Phys. Rev. 37, 405 (1931); 38, 2265 (1931).

29. R. Kubo, M. Toda, N. Hashitsume, Statistical Physics II: Nonequilibrium Statistical Mechanics, 2nd ed. (Springer-Verlag, Berlin, 1991).

30. A. Einstein, Ann. Physik 17, 549 (1905).

31. D. Poilblanc, T. Ziman, J. Bellissard, F. Mila, and G. Montambaux, Europhys. Lett. 22, 537 (1993).

32. P. van Ede van der Pals and P. Gaspard, Phys. Rev. E 49, 79, (1994).

33. For a different illustration of the expansion-contraction picture, see R. van Zon, H. van Beijeren, and J. R. Dorfman, in "Dynamics: Models and Kinetic Methods for Non-equilibrium Many Body Systems", ed. J. Karkheck (Kluwer Academic Publishers, 2000). 